\title{
A Combinatorial Formula for Certain Elements of Upper Cluster Algebras
}

\author{
Kyungyong LEE †๋ $L i L I^{\S}$ and Matthew R. MILLS ${ }^{\dagger}$ \\ $\dagger$ Department of Mathematics, Wayne State University, Detroit, MI 48202, USA \\ E-mail: klee@math.wayne.edu, matthew.mills2@wayne.edu \\ $¥$ Korea Institute for Advanced Study, Seoul, Republic of Korea 130-722 \\ E-mail: klee1@kias.re.kr \\ $\S$ Department of Mathematics and Statistics, Oakland University, Rochester, MI 48309, USA \\ E-mail: li2345@oakland.edu
}

Received September 30, 2014, in final form June 22, 2015; Published online June 26, 2015

http://dx.doi.org/10.3842/SIGMA.2015.049

\begin{abstract}
We develop an elementary formula for certain non-trivial elements of upper cluster algebras. These elements have positive coefficients. We show that when the cluster algebra is acyclic these elements form a basis. Using this formula, we show that each nonacyclic skew-symmetric cluster algebra of rank 3 is properly contained in its upper cluster algebra.
\end{abstract}

Key words: cluster algebra; upper cluster algebra; Dyck path

2010 Mathematics Subject Classification: 13F60

\section{Introduction}

Cluster algebras were introduced by Fomin and Zelevinsky in [5]. A cluster algebra $\mathcal{A}$ is a subalgebra of a rational function field with a distinguished set of generators, called cluster variables, that are generated by an iterative procedure called mutation. By construction cluster variables are rational functions, but it is shown in loc. cit. that they are Laurent polynomials with integer coefficients. Moreover, these coefficients are known to be non-negative [8, 10].

Each cluster algebra $\mathcal{A}$ also determines an upper cluster algebra $\mathcal{U}$, where $\mathcal{A} \subseteq \mathcal{U}$ [4]. It is believed, especially in the context of algebraic geometry, that $\mathcal{U}$ is better behaved than $\mathcal{A}$ (for instance, see $[3,7,8])$. Matherne and Muller [11] gave a general algorithm to compute generators of $\mathcal{U}$. Plamondon $[13,14]$ obtained a (not-necessarily positive) formula for certain elements of skew-symmetric upper cluster algebras using quiver representations. However a directly computable and manifestly positive formula for (non-trivial) elements in $\mathcal{U}$ is not available yet.

In this paper we develop an elementary formula for a family of elements $\{\tilde{x}[\mathbf{a}]\}_{\mathbf{a} \in \mathbb{Z}^{n}}$ of the upper cluster algebra for any fixed initial seed $\Sigma$. We write $\tilde{x}_{\Sigma}[\mathbf{a}]$ for $\tilde{x}[\mathbf{a}]$ when we need to emphasize the dependence on the initial seed $\Sigma$. This family of elements are constructed in Definition 3.1 in terms of sequences of sequences, and an equivalent definition is given in terms of Dyck paths and globally compatible collections in Definition 5.4. One of our main theorems is the following. For the definition of geometric type, see Section 2.

Theorem 1.1. Let $\mathcal{U}$ be the upper cluster algebra of a (not necessarily acyclic) cluster algebra $\mathcal{A}$ of geometric type, and $\Sigma$ be any seed. Then $\tilde{x}_{\Sigma}[\mathbf{a}] \in \mathcal{U}$ for all $\mathbf{a} \in \mathbb{Z}^{n}$.

\footnotetext{
${ }^{\star}$ This paper is a contribution to the Special Issue on New Directions in Lie Theory. The full collection is available at http://www.emis.de/journals/SIGMA/LieTheory2014.html
} 
These elements have some nice properties. They have positive coefficients by definition; they are multiplicative in the sense that we can factorize an element $\tilde{x}[\mathbf{a}]\left(\mathbf{a} \in \mathbb{Z}_{\geq 0}^{n}\right)$ into "elementary pieces" $\tilde{x}\left[\mathbf{a}^{\prime}\right]$ where all entries of $\mathbf{a}^{\prime}$ are 0 or 1 ; for an equioriented quiver of type $A$, these elements form a canonical basis [1]. Moreover, we shall prove in Section 6 the following result. (For the terminology and notation therein, see Section 2.)

Theorem 1.2. Let $\mathcal{A}$ be an acyclic cluster algebra of geometric type, and $\Sigma$ be an acyclic seed. Then $\left\{\tilde{x}_{\Sigma}[\mathbf{a}]\right\}_{\mathbf{a} \in \mathbb{Z}^{n}}$ form a $\mathbb{Z} \mathbb{P}$-basis of $\mathcal{A}$.

For a non-acyclic seed $\Sigma$, the family $\left\{\tilde{x}_{\Sigma}[\mathbf{a}]\right\}$ may neither span $\mathcal{U}$ nor be linearly independent. For a linearly dependent example, see Example 3.2(b). Nevertheless, for certain non-acyclic cluster algebras and for some choice of $\mathbf{a} \in \mathbb{Z}^{n}$, the element $\tilde{x}_{\Sigma}[\mathbf{a}]$ can be used to construct elements in $\mathcal{U} \backslash \mathcal{A}$. One of our main results in this direction is the following:

Theorem 1.3. A non-acyclic rank three skew-symmetric cluster algebra $\mathcal{A}$ of geometric type is not equal to its upper cluster algebra $\mathcal{U}$.

This theorem is inspired by the following results: Berenstein, Fomin and Zelevinsky [4, Proposition 1.26] showed that $\mathcal{A} \neq \mathcal{U}$ for the Markov skew-symmetric matrix $M(2)$, where

$$
M(a)=\left(\begin{array}{ccc}
0 & a & -a \\
-a & 0 & a \\
a & -a & 0
\end{array}\right) .
$$

Speyer [16] found an infinitely generated upper cluster algebra, which is the one associated to the skew-symmetric matrix $M(3)$ with generic coefficients. On the contrary, [11, Proposition 6.2.2] showed that the upper cluster algebra associated to $M(a)$ for $a \geq 2$ but with trivial coefficients is finitely generated, which implies $\mathcal{A} \neq \mathcal{U}$ because $\mathcal{A}$ is known to be infinitely generated [4, Theorem 1.24].

The paper is organized as follows. In Section 2 we review definitions of cluster algebras and upper cluster algebras. Section 3 is devoted to the construction of $\tilde{x}[\mathbf{a}]$ and the proof of Theorem 1.1, and Section 4 to the proof of Theorem 1.3, that $\mathcal{A} \neq \mathcal{U}$ for non-acyclic rank 3 skew-symmetric cluster algebras. Section 5 introduces the Dyck path formula and its relation with the construction in Section 3. Finally, in Section 6, we present two proofs of Theorem 1.2.

\section{Cluster algebras and upper cluster algebras}

Let $m, n$ be positive integers such that $m \geq n$. Denote $\mathcal{F}=\mathbb{Q}\left(x_{1}, \ldots, x_{m}\right)$. A seed $\Sigma=(\tilde{\mathbf{x}}, \tilde{B})$ is a pair where $\tilde{\mathbf{x}}=\left\{x_{1}, \ldots, x_{m}\right\}$ is an $m$-tuple of elements of $\mathcal{F}$ that form a free generating set, $\tilde{B}$ is an $m \times n$ integer matrix such that the submatrix $B$ (called the principal part) formed by the top $n$ rows is sign-skew-symmetric (that is, either $b_{i j}=b_{j i}=0$, or else $b_{j i}$ and $b_{i j}$ are of opposite sign; in particular, $b_{i i}=0$ for all $i$ ). The integer $n$ is called the rank of the seed.

For any integer $a$, let $[a]_{+}:=\max (0, a)$. Given a seed $(\tilde{\mathbf{x}}, \tilde{B})$ and a specified index $1 \leq k \leq n$, we define mutation of $(\tilde{\mathbf{x}}, \tilde{B})$ at $k$, denoted $\mu_{k}(\tilde{\mathbf{x}}, \tilde{B})$, to be a new seed $\left(\tilde{\mathbf{x}}^{\prime}, \tilde{B}^{\prime}\right)$, where

$$
\begin{aligned}
& x_{i}^{\prime}= \begin{cases}x_{k}^{\prime}=x_{k}^{-1}\left(\prod_{i=1}^{m} x_{i}^{\left[b_{i k}\right]_{+}}+\prod_{i=1}^{m} x_{i}^{\left[-b_{i k}\right]_{+}}\right), & \text {if } i=k, \\
x_{i}, & \text { otherwise, }\end{cases} \\
& b_{i j}^{\prime}= \begin{cases}-b_{i j}, & \text { if } i=k \text { or } j=k, \\
b_{i j}+\frac{\left|b_{i k}\right| b_{k j}+b_{i k}\left|b_{k j}\right|}{2}, & \text { otherwise. }\end{cases}
\end{aligned}
$$


If the principal part of $\tilde{B}^{\prime}$ is also sign-skew-symmetric, we say that the mutation is well-defined. Note that a well-defined mutation is an involution, that is mutating $\left(\tilde{\mathbf{x}}^{\prime}, \tilde{B}^{\prime}\right)$ at $k$ will return to our original seed $(\tilde{\mathbf{x}}, \tilde{B})$.

Two seeds $\Sigma_{1}$ and $\Sigma_{2}$ are said to be mutation-equivalent or in the same mutation class if $\Sigma_{2}$ can be obtained by a sequence of well-defined mutations from $\Sigma_{1}$. This is obviously an equivalence relation. A seed $\Sigma$ is said to be totally mutable if every sequence of mutations from $\Sigma$ consists of well-defined ones. It is shown in [5, Proposition 4.5] that a seed is totally mutable if $B$ is skew-symmetrizable, that is, if there exists a diagonal matrix $D$ with positive diagonal entries such that $D B$ is skew-symmetric.

To emphasize the different roles played by $x_{i}(i \leq n)$ and $x_{i}(i>n)$, we also use $(\mathbf{x}, \mathbf{y}, B)$ to denote the seed $(\tilde{\mathbf{x}}, \tilde{B})$, where $\mathbf{x}=\left\{x_{1}, \ldots, x_{n}\right\}, \mathbf{y}=\left\{y_{1}, \ldots, y_{n}\right\}$ where $y_{j}=\prod_{i=n+1}^{m} x_{i}^{b_{i j}}$. We call $\mathbf{x}$ a cluster, $\mathbf{y}$ a coefficient tuple, $B$ the exchange matrix, and the elements of a cluster cluster variables. We denote

$$
\mathbb{Z} \mathbb{P}=\mathbb{Z}\left[x_{n+1}^{ \pm 1}, \ldots, x_{m}^{ \pm 1}\right]
$$

In the paper, we shall only study cluster algebras of geometric type, defined as follows.

Definition 2.1. Given a totally mutable seed $(\mathbf{x}, \mathbf{y}, B)$, the cluster algebra $\mathcal{A}(\mathbf{x}, \mathbf{y}, B)$ of geometric type is the subring of $\mathcal{F}$ generated over $\mathbb{Z} \mathbb{P}$ by

$$
\bigcup_{\left(\mathbf{x}^{\prime}, \mathbf{y}^{\prime}, B^{\prime}\right)} \mathbf{x}^{\prime}
$$

where the union runs over all seeds $\left(\mathbf{x}^{\prime}, \mathbf{y}^{\prime}, B^{\prime}\right)$ that are mutation-equivalent to $(\mathbf{x}, \mathbf{y}, B)$. The seed $(\mathbf{x}, \mathbf{y}, B)$ is called the initial seed of $\mathcal{A}(\mathbf{x}, \mathbf{y}, B)$. (Since $(\mathbf{x}, \mathbf{y}, B)=(\tilde{\mathbf{x}}, \tilde{B})$ in our notation, $\mathcal{A}(\mathbf{x}, \mathbf{y}, B)$ is also denoted $\mathcal{A}(\tilde{\mathbf{x}}, \tilde{B})$.)

It follows from the definition that any seed in the same mutation class will generate the same cluster algebra up to isomorphism.

For any $n \times n$ sign-skew-symmetric matrix $B$, we associate a (simple) directed graph $Q_{B}$ with vertices $1, \ldots, n$, such that for each pair $(i, j)$ with $b_{i j}>0$, there is exactly one arrow from vertex $i$ to vertex $j$. (Note that even if $B$ is skew-symmetric, $Q_{B}$ is not the usual quiver associated to $B$ which can have multiple edges.)

We call $B$ (as well as the digraph $Q_{B}$ and the seed $\Sigma=(\mathbf{x}, \mathbf{y}, B)$ ) acyclic if there are no oriented cycles in $Q_{B}$. We say that the cluster algebra $\mathcal{A}(\mathbf{x}, \mathbf{y}, B)$ is acyclic if there exists an acyclic seed; otherwise we say that the cluster algebra is non-acyclic.

Definition 2.2. Given a cluster algebra $\mathcal{A}$, the upper cluster algebra $\mathcal{U}$ is defined as

$$
\mathcal{U}=\bigcap_{\mathbf{x}=\left\{x_{1}, \ldots, x_{n}\right\}} \mathbb{Z} \mathbb{P}\left[x_{1}^{ \pm 1}, \ldots, x_{n}^{ \pm 1}\right]
$$

where x runs over all clusters of $\mathcal{A}$.

Now we can give the following definition of coprime when the cluster algebra is of geometric type (given in [4, Lemma 3.1]).

Definition 2.3. A seed $(\tilde{\mathbf{x}}, \tilde{B})$ is coprime if no two columns of $\tilde{B}$ are proportional to each other with the proportionality coefficient being a ratio of two odd integers.

A cluster algebra is totally coprime if every seed is coprime. 
In certain cases it is sufficient to consider only the clusters of the initial seed and the seeds that are a single mutation away from it, rather than all the seeds in the entire mutation class. For a cluster $\mathbf{x}$, let $\mathcal{U}_{\mathbf{x}}$ be the intersection in $\mathbb{Z} \mathbb{P}\left(x_{1}, \ldots, x_{n}\right)$ of the $n+1$ Laurent rings corresponding to $\mathbf{x}$ and its one-step mutations:

$$
\mathcal{U}_{\mathbf{x}}:=\mathbb{Z} \mathbb{P}\left[x_{1}^{ \pm 1}, \ldots, x_{n}^{ \pm 1}\right] \cap\left(\bigcap_{i} \mathbb{Z} \mathbb{P}\left[x_{1}^{ \pm 1}, \ldots, x_{i}^{\prime \pm 1}, \ldots, x_{n}^{ \pm 1}\right]\right) .
$$

Theorem 2.4 ([4, 12]). We have $\mathcal{A} \subseteq \mathcal{U} \subseteq \mathcal{U}_{\mathbf{x}}$. Moreover,

(i) If $\mathcal{A}$ is acyclic, then $\mathcal{A}=\mathcal{U}$.

(ii) If $\mathcal{A}$ is totally coprime, then $\mathcal{U}=\mathcal{U}_{\mathbf{x}}$ for any seed $(\mathbf{x}, \mathbf{y}, B)$. In particular, this holds when the matrix $\tilde{B}$ has full rank.

\section{Construction of some elements in the upper cluster algebra}

Fix an initial seed $\Sigma$ (thus $\tilde{B}$ is fixed). In this section, we construct Laurent polynomials $\tilde{x}[\mathbf{a}]$ $\left(=\tilde{x}_{\Sigma}[\mathbf{a}]\right)$ and show that they are in the upper cluster algebra.

We define $b_{i j}=-b_{j i}$ for $1 \leq i \leq n, n+1 \leq j \leq m$, and define $b_{i j}=0$ if $i, j>n$. Define

$$
Q_{\tilde{B}}=\left\{(i, j) \mid 1 \leq i, j \leq m, b_{i j}>0\right\} .
$$

By abuse of notation we also use $Q_{\tilde{B}}$ to denote the digraph with vertex set $\{1, \ldots, m\}$ and edge set $Q_{\tilde{B}}$. Then $Q_{B}$ is a full sub-digraph of $Q_{\tilde{B}}$ that consists of the first $n$ vertices.

We define the following operations on the set of finite $\{0,1\}$-sequences. Let $t=\left(t_{1}, \ldots, t_{a}\right)$, $t^{\prime}=\left(t_{1}^{\prime}, \ldots, t_{b}^{\prime}\right)$. Define

$$
\bar{t}=\left(\bar{t}_{1}, \ldots, \bar{t}_{a}\right)=\left(1-t_{1}, \ldots, 1-t_{a}\right), \quad|t|=\sum_{r=1}^{a} t_{r}, \quad t \cdot t^{\prime}=\sum_{r=1}^{\min (a, b)} t_{r} t_{r}^{\prime} .
$$

and for $t=() \in\{0,1\}^{0}$, define $\bar{t}=()$.

Definition 3.1. Let $\mathbf{a}=\left(a_{i}\right) \in \mathbb{Z}^{n}$.

(i) Let $\mathbf{s}=\left(\mathbf{s}_{1}, \ldots, \mathbf{s}_{n}\right)$ where $\mathbf{s}_{i}=\left(s_{i, 1}, s_{i, 2}, \ldots, s_{i,\left[a_{i}\right]_{+}}\right) \in\{0,1\}^{\left[a_{i}\right]_{+}}$for $i=1, \ldots, n$. Let $S_{\text {all }}=S_{\text {all }}(\mathbf{a})$ be the set of all such $\mathbf{s}$. Let $S_{\mathrm{gcc}}=S_{\mathrm{gcc}}(\mathbf{a})=\left\{\mathbf{s} \in S_{\text {all }} \mid \mathbf{s}_{i} \cdot \overline{\mathbf{s}}_{j}=0\right.$ for every $\left.(i, j) \in Q_{B}\right\}$.

By convention, we assume that $a_{i}=0$ and $\mathbf{s}_{i}=()$ for $i>n$.

(ii) Define $\tilde{x}[\mathbf{a}]\left(=\tilde{x}_{\Sigma}[\mathbf{a}]\right)$ to be the Laurent polynomial

$$
\tilde{x}[\mathbf{a}]:=\left(\prod_{l=1}^{n} x_{l}^{-a_{l}}\right) \sum_{\mathbf{s} \in S_{\mathrm{gcc}}}\left(\prod_{(i, j) \in Q_{\tilde{B}}} x_{i}^{b_{i j}\left|\overline{\mathbf{s}}_{j}\right|} x_{j}^{-b_{j i}\left|\mathbf{s}_{i}\right|}\right) .
$$

(Note that the exponent $-b_{j i}\left|\mathbf{s}_{i}\right|$ is nonnegative since $b_{j i}<0$ for $(i, j) \in Q_{\tilde{B}}$.)

(iii) Define $z[\mathbf{a}]\left(=z_{\Sigma}[\mathbf{a}]\right)$ to be the Laurent polynomial

$$
z[\mathbf{a}]:=\left(\prod_{l=1}^{n} x_{l}^{-a_{l}}\right) \sum_{\mathbf{s} \in S_{\text {all }}}\left(\prod_{(i, j) \in Q_{\tilde{B}}} x_{i}^{b_{i j}\left|\overline{\mathbf{s}}_{j}\right|} x_{j}^{-b_{j i}\left|\mathbf{s}_{i}\right|}\right) .
$$


Example 3.2. (a) Use Definition 3.1 to compute $\tilde{x}[\mathbf{a}]$ for $n=2, m=3$, $\mathbf{a}=(1,1)$, and

$$
\tilde{B}=\left[\begin{array}{cc}
0 & a \\
-a^{\prime} & 0 \\
c & -b
\end{array}\right], \quad a, a^{\prime}, b, c>0 .
$$

Then we have $Q_{\tilde{B}}=\{(1,2),(2,3),(3,1)\}, S_{\mathrm{gcc}}=\{((0),(0)),((0),(1)),((1),(1))\}$. Note that $\mathbf{s}=((1),(0))$ is not in $S_{\text {gcc }}$ because $(1,2) \in Q_{\tilde{B}}$ but $\mathbf{s}_{1} \cdot \overline{\mathbf{s}}_{2}=1(1-0)=1 \neq 0$. Thus

$$
\begin{aligned}
\tilde{x}[\mathbf{a}] & =x_{1}^{-1} x_{2}^{-1}\left(\left(x_{1}^{a} x_{2}^{0}\right)\left(x_{2}^{0} x_{3}^{0}\right)\left(x_{3}^{c} x_{1}^{0}\right)+\left(x_{1}^{0} x_{2}^{0}\right)\left(x_{2}^{0} x_{3}^{b}\right)\left(x_{3}^{c} x_{1}^{0}\right)+\left(x_{1}^{0} x_{2}^{a^{\prime}}\right)\left(x_{2}^{0} x_{3}^{b}\right)\left(x_{3}^{0} x_{1}^{0}\right)\right) \\
& =x_{1}^{-1} x_{2}^{-1}\left(x_{1}^{a} x_{3}^{c}+x_{3}^{b} x_{3}^{c}+x_{2}^{a^{\prime}} x_{3}^{b}\right) .
\end{aligned}
$$

(b) For a non-acyclic seed, $\tilde{x}[\mathbf{a}]$ is less interesting for certain choices of a: take $n=m=3$, $\mathbf{a}=(1,1,1)$ and

$$
\tilde{B}=\left[\begin{array}{ccc}
0 & a & -c^{\prime} \\
-a^{\prime} & 0 & b \\
c & -b^{\prime} & 0
\end{array}\right], \quad a, a^{\prime}, b, b^{\prime}, c, c^{\prime}>0
$$

Then $Q_{\tilde{B}}=\{(1,2),(2,3),(3,1)\}, S_{\mathrm{gcc}}=\{((0),(0),(0)),((1),(1),(1))\}$. Thus

$$
\tilde{x}[\mathbf{a}]=\frac{x_{1}^{a} x_{2}^{b} x_{3}^{c}+x_{2}^{a^{\prime}} x_{3}^{b^{\prime}} x_{1}^{c^{\prime}}}{x_{1} x_{2} x_{3}}
$$

which can be reduced to $x_{1}^{a-1} x_{2}^{b-1} x_{3}^{c-1}+x_{2}^{a^{\prime}-1} x_{3}^{b^{\prime}-1} x_{1}^{c^{\prime}-1}$, that is

$$
\tilde{x}[1,1,1]=\tilde{x}[(1-a, 1-b, 1-c)]+\tilde{x}\left[\left(1-a^{\prime}, 1-b^{\prime}, 1-c^{\prime}\right)\right] .
$$

Thus $\{\tilde{x}[\mathbf{a}]\}$ is not $\mathbb{Z} \mathbb{P}$-linear independent.

Lemma 3.3 (multiplicative property of $\tilde{x}[\mathbf{a}]$ and $z[\mathbf{a}]$ ). Fix a seed $\Sigma$.

(i) For $k, a \in \mathbb{Z}, \mathbf{a} \in \mathbb{Z}^{n}$, define

$$
\begin{gathered}
f_{k}(a):= \begin{cases}1, & \text { if } k \leq a, \\
0, & \text { otherwise, }\end{cases} \\
f_{k}(\mathbf{a}):=\left(f_{k}\left(a_{1}\right), \ldots, f_{k}\left(a_{n}\right)\right) \in\{0,1\}^{n}, \mathbf{a}_{+}:=\left(\left[a_{1}\right]_{+},\left[a_{2}\right]_{+}, \ldots,\left[a_{n}\right]_{+}\right) . \text {Then } \\
\tilde{x}[\mathbf{a}]=\left(\prod_{i=1}^{n} x_{i}^{\left[-a_{i}\right]_{+}}\right) \tilde{x}\left[\mathbf{a}_{+}\right]=\left(\prod_{i=1}^{n} x_{i}^{\left[-a_{i}\right]_{+}}\right) \prod_{k \geq 1} \tilde{x}\left[f_{k}\left(\mathbf{a}_{+}\right)\right] .
\end{gathered}
$$

(ii) Assume that the underlying undirected graph of $Q_{B}$ has $c>1$ components, inducing a partition of the vertex set $\{1, \ldots, n\}=I_{1} \cup \cdots \cup I_{c}$. Define $\mathbf{a}^{(j)}=\left(a_{1}^{(j)}, \ldots, a_{c}^{(j)}\right)$ by $a_{i}^{(j)}=a_{i}$ if $i \in I_{j}$, otherwise $a_{i}^{(j)}=0$. Then

$$
\tilde{x}[\mathbf{a}]=\prod_{j=1}^{c} \tilde{x}\left[\mathbf{a}^{(j)}\right] .
$$

(Note that each factor $\tilde{x}\left[\mathbf{a}^{(j)}\right]$ can be regarded as an element in a cluster algebra of rank $\left|I_{j}\right|<n$, with the same $\mathbb{Z} \mathbb{P}$.) 
(iii) We have $z[\mathbf{a}]=\prod_{i=1}^{n} x_{i}^{\left\langle-a_{i}\right\rangle}$ where we use the notation

$$
x_{i}^{\langle r\rangle}= \begin{cases}x_{i}^{r}, & \text { if } r \geq 0, \\ \left(x_{i}^{\prime}\right)^{-r}, & \text { if } r<0 .\end{cases}
$$

(Recall that $x_{i}^{\prime}$ is obtained by mutating the initial seed at $\left.i\right)$. As a consequence, $(i),(i i)$ still hold if we replace $\tilde{x}[-]$ by $z[-]$. Moreover, if the seed $\Sigma$ is acyclic, then $\{z[\mathbf{a}]\} \mathbf{a} \in \mathbb{Z}^{n}$ is the standard monomial basis, i.e., the set of monomials in $x_{1}, \ldots, x_{n}, x_{1}^{\prime}, \ldots, x_{n}^{\prime}$ which contain no product of the form $x_{j} x_{j}^{\prime}$.

Proof. (i) The first equality is obvious. For the second equality, assuming $\mathbf{a}=\mathbf{a}_{+}$. For a sequence $t=\left(t_{1}, \ldots, t_{a}\right) \in\{0,1\}^{a}$, we can regard it as an infinite sequence $\left(t_{1}, \ldots, t_{a}, 0,0, \ldots\right)$. Then $\bar{t}=\left(f_{r}(a)-t_{r}\right)_{r=1}^{\infty}$. The sum and dot product in (3.1) extend naturally.

Then $\tilde{x}[\mathbf{a}]$ is the coefficient of $z^{0}$ in the following polynomial in $\mathbb{Z}\left[x_{1}^{ \pm 1}, \ldots, x_{m}^{ \pm 1}\right][z]$ (note that all the products $\prod_{k \geq 1}$ appearing below are finite products since the factors are 1 if $k>$ $\left.\max \left(\left[a_{1}\right]_{+}, \ldots,\left[a_{n}\right]_{+}\right)\right)$

$$
\begin{aligned}
\left(\prod_{i=1}^{n} x_{i}^{-a_{i}}\right) \sum_{\mathbf{s} \in S_{\text {all }}}\left(\prod_{(i, j) \in Q_{\tilde{B}}} x_{i}^{b_{i j}\left|\overline{\mathbf{s}}_{j}\right|} x_{j}^{-b_{j i}\left|\mathbf{s}_{i}\right|} z^{\mathbf{s}_{i} \cdot \overline{\mathbf{s}}_{j}}\right) \\
=\left(\prod_{i=1}^{n} x_{i}^{-a_{i}}\right) \sum_{\mathbf{s} \in S_{\text {all }}} \prod_{k \geq 1}\left(\prod_{(i, j) \in Q_{\tilde{B}}} x_{i}^{b_{i j} \bar{s}_{j, k}} x_{j}^{-b_{j i} s_{i, k}} z^{s_{i, k} \bar{s}_{j, k}}\right) \\
=\left(\prod_{i=1}^{n} x_{i}^{-a_{i}}\right) \sum_{\mathbf{s} \in S_{\text {all }}} \prod_{k \geq 1}\left(\prod_{(i, j) \in Q_{\tilde{B}}} x_{i}^{b_{i j}\left(f_{k}\left(a_{j}\right)-s_{j, k}\right)} x_{j}^{-b_{j i} s_{i, k}} z^{s_{i, k}\left(f_{k}\left(a_{j}\right)-s_{j, k}\right)}\right) \\
=\left(\prod_{i=1}^{n} x_{i}^{-a_{i}}\right) \prod_{k \geq 1}\left(\sum_{\mathbf{s}^{k} \in S_{\text {all }}^{k}} \prod_{(i, j) \in Q_{\tilde{B}}} x_{i}^{b_{i j}\left(f_{k}\left(a_{j}\right)-s_{j, k}\right)} x_{j}^{-b_{j i} s_{i, k}} z^{s_{i, k}\left(f_{k}\left(a_{j}\right)-s_{j, k}\right)}\right),
\end{aligned}
$$

where $S_{\text {all }}^{k}$ is the set of all possible $\mathbf{s}^{k}=\left(s_{1, k}, \ldots, s_{n, k}\right)$ with $\left(\mathbf{s}_{1}, \ldots, \mathbf{s}_{m}\right)$ running through $S_{\text {all }}$ (recall that $s_{i, k}$ is the $k$-th number of $\mathbf{s}_{i}$, and by convention $s_{i, k}=0$ if $k>\left[a_{i}\right]_{+}$). Equivalently,

$$
S_{\text {all }}^{k}=\left\{\mathbf{s}^{k}=\left(s_{1, k}, \ldots, s_{n, k}\right) \in\{0,1\}^{n} \mid 0 \leq s_{i, k} \leq f_{k}\left(a_{i}\right) \text { for } i=1, \ldots, n\right\} .
$$

Meanwhile, denote $f_{k}(\mathbf{a})=\left(f_{k}\left(a_{1}\right), \ldots, f_{k}\left(a_{n}\right)\right) \in\{0,1\}^{n}$. Then $\tilde{x}\left[f_{k}(\mathbf{a})\right]$ is the coefficient of $z^{0}$ of

$$
\left(\prod_{i=1}^{n} x_{i}^{-f_{k}\left(a_{i}\right)}\right) \sum_{\mathbf{s}^{k} \in S_{\text {all }}^{k}}\left(\prod_{(i, j) \in Q_{\tilde{B}}} x_{i}^{b_{i j}\left(f_{k}\left(a_{j}\right)-s_{j, k}\right)} x_{j}^{-b_{j i} s_{i, k}} z^{s_{i, k}\left(f_{k}\left(a_{j}\right)-s_{j, k}\right)}\right) .
$$

So we conclude that

$$
\begin{aligned}
\tilde{x}[\mathbf{a}] & =\left(\prod_{i=1}^{n} x_{i}^{-a_{i}}\right) \prod_{k \geq 1}\left(\tilde{x}\left[f_{k}(\mathbf{a})\right] \prod_{i=1}^{n} x_{i}^{f_{k}\left(a_{i}\right)}\right) \\
& =\left(\prod_{i=1}^{n} x_{i}^{-a_{i}+\sum_{k \geq 1} f_{k}\left(a_{i}\right)}\right) \prod_{k \geq 1} \tilde{x}\left[f_{k}(\mathbf{a})\right]=\prod_{k \geq 1} \tilde{x}\left[f_{k}(\mathbf{a})\right] .
\end{aligned}
$$


(ii) There is a bijection

$$
\prod_{j=1}^{c} S_{\mathrm{gcc}}\left(\mathbf{a}^{(j)}\right) \rightarrow S_{\mathrm{gcc}}(\mathbf{a}), \quad\left(\mathbf{s}^{(1)}, \ldots, \mathbf{s}^{(c)}\right) \mapsto \mathbf{s}=\left(\mathbf{s}_{1}, \ldots, \mathbf{s}_{n}\right),
$$

where $\mathbf{s}_{i}=\mathbf{s}_{i}^{(j)}$ if $i \in I_{j}$. This bijection induces the expected equality.

(iii) Rewrite

$$
\begin{aligned}
z[\mathbf{a}] & =\left(\prod_{i=1}^{n} x_{i}^{-a_{i}}\right) \sum_{\mathbf{s}_{1}, \ldots, \mathbf{s}_{n}}\left(\prod_{i, j} x_{i}^{\left|\overline{\mathbf{s}}_{j}\right|\left[b_{i j}\right]_{+}} x_{j}^{\left|\mathbf{s}_{i}\right|\left[-b_{j i}\right]_{+}}\right) \\
& =\sum_{\mathbf{s}_{1}, \ldots, \mathbf{s}_{n}} \prod_{k=1}^{n}\left(x_{k}^{-a_{k}} \prod_{i} x_{i}^{\left|\mathbf{s}_{k}\right|\left[b_{i k}\right]_{+}} \prod_{j} x_{j}^{\left|\mathbf{s}_{k}\right|\left[-b_{j k}\right]_{+}}\right) \\
& =\prod_{k=1}^{n} x_{k}^{-a_{k}} \sum_{\mathbf{s}_{k}}\left(\prod_{i} x_{i}^{\left|\overline{\mathbf{s}}_{k}\right|\left[b_{i k}\right]+} \prod_{j} x_{j}^{\left|\mathbf{s}_{k}\right|\left[-b_{j k}\right]_{+}}\right)=\prod_{k=1}^{n} z\left[a_{k} e_{k}\right] .
\end{aligned}
$$

If $a_{k} \leq 0$, then $\mathbf{s}_{k}=()$, therefore $z\left[a_{k} e_{k}\right]=x_{k}^{-a_{k}}=x_{k}^{\left\langle-a_{k}\right\rangle}$. If $a_{k}>0$, then

$$
\begin{aligned}
z\left[a_{k} e_{k}\right] & =x_{k}^{-a_{k}} \sum_{s_{k, 1}, \ldots, s_{k, a_{k}}}\left(\prod_{i} x_{i}^{\sum^{a_{k}=1}\left(1-s_{k, r}\right)\left[b_{i k}\right]_{+}} \prod_{j} x_{j}^{\sum_{k=1}^{a_{k}}\left(s_{k, r}\right)\left[-b_{j k}\right]_{+}}\right) \\
& =x_{k}^{-a_{k}} \prod_{r=1}^{a_{k}} \sum_{s_{k, r} \in\{0,1\}}\left(\prod_{i} x_{i}^{\left(1-s_{k, r}\right)\left[b_{i k}\right]_{+}} \prod_{j} x_{j}^{\left(s_{k, r}\right)\left[-b_{j k}\right]_{+}}\right) \\
& =x_{k}^{-a_{k}} \prod_{r=1}^{a_{k}}\left(\prod_{i} x_{i}^{\left[b_{i k}\right]_{+}}+\prod_{j} x_{j}^{\left[-b_{j k}\right]_{+}}\right)=\left(x_{k}^{\prime}\right)^{a_{k}}=x_{k}^{\left\langle-a_{k}\right\rangle} .
\end{aligned}
$$

This proves $z[\mathbf{a}]=\prod_{i=1}^{n} x_{i}^{\left\langle-a_{i}\right\rangle}$. The analogue of (i), (ii) immediately follows. The fact that $\{z[\mathbf{a}]\}_{\mathbf{a} \in \mathbb{Z}^{n}}$ forms a basis is proved in [4, Theorem 1.16].

Remark 3.4. For readers who are familiar with [1, Lemma 4.2], they may notice that the decomposition therein is similar to Lemma 3.3(i) above. Indeed, [1, Lemma 4.2] gives a finer decomposition. For example, for the coefficient-free cluster algebra of the quiver $1 \rightarrow 2 \rightarrow 3$, $\tilde{x}[(2,1,3)]$ will decompose as $\tilde{x}[(1,1,1)] \tilde{x}[(1,0,1)] \tilde{x}[(0,0,1)]$ in Lemma 3.3(i), but decompose as $\tilde{x}[(1,1,1)] \tilde{x}[(1,0,0)] \tilde{x}[(0,0,1)]^{2}$ in $[1$, Lemma 4.2$]$.

The following lemma focuses on the case where $\mathbf{a}=\left(a_{i}\right) \in\{0,1\}^{n}$ as opposed to that in $\mathbb{Z}^{n}$. The condition of $S_{\mathrm{gcc}}$ takes a much simpler form: we can treat sequences (0) and (1) as numbers 0 and 1 respectively, and the condition $\mathbf{s}_{i} \cdot \overline{\mathbf{s}}_{j}=0$ can be written as $\left(s_{i}, a_{j}-s_{j}\right) \neq(1,1)$. We shall use $\mathbf{S}$ to denote this simpler form of $S_{\text {gcc }}$.

Lemma 3.5. For $\mathbf{a}=\left(a_{i}\right) \in\{0,1\}^{n}$, denote by $\mathbf{S}$ the set of all $n$-tuples $\mathbf{s}=\left(s_{1}, \ldots, s_{n}\right) \in\{0,1\}^{n}$ such that $0 \leq s_{i} \leq a_{i}$ for $i=1, \ldots, n$, and $\left(s_{i}, a_{j}-s_{j}\right) \neq(1,1)$ for every $(i, j) \in Q_{B}$. By convention we assume $a_{i}=0$ and $s_{i}=0$ if $i>n$. Then 
(i) $\tilde{x}[\mathbf{a}]$ can be written as

$$
\left(\prod_{i=1}^{n} x_{i}^{-a_{i}}\right) \sum_{\mathbf{s} \in \mathbf{S}} \prod_{i=1}^{m} x_{i}^{\sum_{j=1}^{n}\left(a_{j}-s_{j}\right)\left[b_{i j}\right]_{+}+s_{j}\left[-b_{i j}\right]_{+}} .
$$

(ii) $\tilde{x}[\mathbf{a}]$ is in the upper cluster algebra $\mathcal{U}$.

Proof. (i) By Definition 3.1,

$$
\begin{aligned}
\tilde{x}[\mathbf{a}] & =\left(\prod_{i=1}^{n} x_{i}^{-a_{i}}\right) \sum_{\mathbf{s} \in \mathbf{S}}\left(\prod_{(i, j) \in Q_{\tilde{B}}} x_{i}^{\left(a_{j}-s_{j}\right) b_{i j}} x_{j}^{s_{i}\left(-b_{j i}\right)}\right) \\
& =\left(\prod_{i=1}^{n} x_{i}^{-a_{i}}\right) \sum_{\mathbf{s} \in \mathbf{S}}\left(\prod_{i, j=1}^{m} x_{i}^{\left(a_{j}-s_{j}\right)\left[b_{i j}\right]_{+}} x_{j}^{s_{i}\left[-b_{j i}\right]_{+}}\right) \\
& =\left(\prod_{i=1}^{n} x_{i}^{-a_{i}}\right) \sum_{\mathbf{s} \in \mathbf{S}}\left(\prod_{i, j=1}^{m} x_{i}^{\left(a_{j}-s_{j}\right)\left[b_{i j}\right]_{+}}\right)\left(\prod_{i, j=1}^{m} x_{j}^{s_{i}\left[-b_{j i}\right]_{+}}\right) \\
& =\left(\prod_{i=1}^{n} x_{i}^{-a_{i}}\right) \sum_{\mathbf{s} \in \mathbf{S}}\left(\prod_{i, j=1}^{m} x_{i}^{\left(a_{j}-s_{j}\right)\left[b_{i j}\right]_{+}}\right)\left(\prod_{i, j=1}^{m} x_{i}^{s_{j}\left[-b_{i j}\right]_{+}}\right) \\
& =\left(\prod_{i=1}^{n} x_{i}^{-a_{i}}\right) \sum_{\mathbf{s} \in \mathbf{S}}\left(\prod_{i, j=1}^{m} x_{i}^{\left(a_{j}-s_{j}\right)\left[b_{i j}\right]_{+}+s_{j}\left[-b_{i j}\right]_{+}}\right)=(3.2) .
\end{aligned}
$$

(ii) We introduce $n$ extra variables $x_{m+1}, \ldots, x_{m+n}$. Let

$$
\mathbb{Z} \mathbb{P}^{\prime}=\mathbb{Z} \mathbb{P}\left[x_{m+1}^{ \pm 1}, \ldots, x_{m+n}^{ \pm 1}\right]=\mathbb{Z}\left[x_{n+1}^{ \pm 1}, \ldots, x_{m+n}^{ \pm 1}\right]
$$

be the ring of Laurent polynomials in the variables $x_{n+1}, \ldots, x_{m+n}$. Let

$$
\tilde{B}^{\prime}=\left[\begin{array}{c}
\tilde{B} \\
I_{n}
\end{array}\right]
$$

be the $(m+n) \times n$ matrix that encodes a new cluster algebra $\mathcal{A}^{\prime}$. Assume $a_{n+1}=\cdots=a_{m+n}=0$ and define $Q_{\tilde{B}}^{\prime}, \mathbf{S}^{\prime}, \tilde{x}^{\prime}[\mathbf{a}]$ for $\mathbb{Z} \mathbb{P}^{\prime}$ similarly as the definition of $Q_{\tilde{B}}, \mathbf{S}, \tilde{x}[\mathbf{a}]$ for $\mathbb{Z} \mathbb{P}$. (Of course $\mathbf{S}^{\prime}=\mathbf{S}$, but we use different notation to emphasize that they are for different cluster algebras). So

$$
\tilde{x}^{\prime}[\mathbf{a}]=\left(\prod_{i=1}^{n} x_{i}^{-a_{i}}\right) \sum_{\mathbf{s} \in \mathbf{S}^{\prime}} P_{\mathbf{s}}, \quad P_{\mathbf{s}}:=\prod_{i=1}^{m+n} x_{i}^{\sum_{j=1}^{n}\left(a_{j}-s_{j}\right)\left[b_{i j}\right]_{+}+s_{j}\left[-b_{i j}\right]_{+}} .
$$

We will show that $\tilde{x}^{\prime}[\mathbf{a}]$ is in the upper bound

$$
\mathcal{U}_{x}^{\prime}=\mathbb{Z} \mathbb{P}^{\prime}\left[\mathbf{x}^{ \pm 1}\right] \cap \mathbb{Z} \mathbb{P}^{\prime}\left[\mathbf{x}_{1}^{ \pm 1}\right] \cap \cdots \cap \mathbb{Z P}^{\prime}\left[\mathbf{x}_{n}^{ \pm 1}\right]
$$

(where $\mathbf{x}=\left\{x_{1}, \ldots, x_{n}\right\}$ and for $1 \leq k \leq n$, the adjacent cluster $\mathbf{x}_{k}$ is defined by $\mathbf{x}_{k}=$ $\mathbf{x}-\left\{x_{k}\right\} \cup\left\{x_{k}^{\prime}\right\}$ ), therefore $\tilde{x}^{\prime}[\mathbf{a}]$ is in the upper cluster algebra $\mathcal{U}^{\prime}$, thanks to the fact that $\mathcal{U}_{x}^{\prime}=\mathcal{U}^{\prime}$ when $\tilde{B}^{\prime}$ is of full rank [4, Corollary 1.7 and Proposition 1.8]. Then we substitute $x_{n+1}=\cdots=x_{m+n}=1$ and conclude that $\tilde{x}[\mathbf{a}]$ is in the upper cluster algebra $\mathcal{U}$. 
Since $\tilde{x}^{\prime}[\mathbf{a}]$ is obviously in $\mathbb{Z} \mathbb{P}^{\prime}\left[\mathbf{x}^{ \pm 1}\right]$ from its definition, we only need to show that $\tilde{x}^{\prime}[\mathbf{a}]$ is in $\mathbb{Z} \mathbb{P}^{\prime}\left[\mathbf{x}_{k}^{ \pm 1}\right]$ for $1 \leq k \leq n$. Again from its definition we see that $\tilde{x}^{\prime}[\mathbf{a}]$ is in $\mathbb{Z} \mathbb{P}^{\prime}\left[\mathbf{x}_{k}^{ \pm 1}\right]$ when $a_{k}=0$. So we may assume $a_{k}=1$. Let $N \subset \mathbf{S}^{\prime}$ contain those $\mathbf{s}$ such that $P_{\mathbf{s}}$ is not divisible by $x_{k}$; equivalently,

$$
N=\left\{\mathbf{s} \in \mathbf{S}^{\prime} \mid s_{j}=a_{j} \text { if } b_{k j}>0 ; s_{j}=0 \text { if } b_{k j}<0\right\} .
$$

Then it suffices to show that $\sum_{\mathbf{s} \in N} P_{\mathbf{s}}$ is divisible by $A$, where

$$
A=x_{k}^{\prime} x_{k}=\prod_{i=1}^{m+n} x_{i}^{\left[b_{i k}\right]_{+}}+\prod_{i=1}^{m+n} x_{i}^{\left[-b_{i k}\right]_{+}} .
$$

Write $N$ into a partition $N=N_{0} \cup N_{1}$ where $N_{0}=\left\{\mathbf{s} \in N \mid s_{k}=0\right\}, N_{1}=\left\{\mathbf{s} \in N \mid s_{k}=1\right\}$. Define $\varphi: N_{0} \rightarrow N_{1}$ by

$$
\varphi\left(s_{1}, \ldots, s_{k-1}, 0, s_{k+1}, \ldots, s_{n}\right)=\left(s_{1}, \ldots, s_{k-1}, 1, s_{k+1}, \ldots, s_{n}\right) .
$$

Then $\varphi$ is a well-defined bijection because in the definition of $N$ there is no condition imposed on $s_{k}$. Thus

$$
\begin{aligned}
\sum_{\mathbf{s} \in N} P_{\mathbf{s}} & =\sum_{\mathbf{s} \in N_{0}} P_{\mathbf{s}}+\sum_{\mathbf{s} \in N_{1}} P_{\mathbf{s}}=\sum_{\mathbf{s} \in N_{0}}\left(P_{\mathbf{s}}+P_{\varphi(\mathbf{s})}\right) \\
& =\prod_{i=1}^{m+n} \prod_{\substack{1 \leq j \leq n \\
j \neq k}} x_{i}^{\left(a_{j}-s_{j}\right)\left[b_{i j}\right]_{+}+s_{j}\left[-b_{i j}\right]_{+}}\left(\prod_{i=1}^{m+n} x_{i}^{\left[b_{i k}\right]_{+}}+\prod_{i=1}^{m+n} x_{i}^{\left[-b_{i k}\right]_{+}}\right) \\
& =\prod_{i=1}^{m+n} \prod_{\substack{1 \leq j \leq n \\
j \neq k}} x_{i}^{\left(a_{j}-s_{j}\right)\left[b_{i j}\right]_{+}+s_{j}\left[-b_{i j}\right]_{+}} A
\end{aligned}
$$

is divisible by $A$.

Below is the main theorem of the section.

Proof of Theorem 1.1. It follows immediately from Lemma 3.3(i) and Lemma 3.5(ii). Indeed, we use Lemma 3.3(i) to factor $\tilde{x}[\mathbf{a}]$ (for $\mathbf{a} \in \mathbb{Z}_{\geq 0}^{n}$ ) into the product of a usual monomial (which is in $\mathcal{U}$ ) and those $\tilde{x}\left[\mathbf{a}^{\prime}\right]$ 's where all entries of $\mathbf{a}^{\prime}$ are 0 or 1 (which is also in $\mathcal{U}$ by Lemma 3.5(ii).

Remark 3.6. We claim that if $\Sigma$ is acyclic (i.e., $Q_{B}$ is acyclic), then $\left(\prod_{i=1}^{n} x_{i}^{a_{i}}\right) \tilde{x}[\mathbf{a}]$ is not divisible by $x_{k}$ for any $1 \leq k \leq n$, i.e., there exists $\mathbf{s}=\left(\mathbf{s}_{1}, \ldots, \mathbf{s}_{n}\right) \in S_{\text {gcc }}$ such that

$$
\prod_{(i, j) \in Q_{\tilde{B}}} x_{i}^{b_{i j}\left|\overline{\mathbf{s}}_{j}\right|} x_{j}^{-b_{j i}\left|\mathbf{s}_{i}\right|}
$$

is not divisible by $x_{k}$. Fix $k$ such that $1 \leq k \leq n$. We need to find $\mathbf{s}$ such that

$$
\left|\overline{\mathbf{s}}_{j}\right|=0 \quad \text { if } \quad(k, j) \in Q_{\tilde{B}} \quad \text { and } \quad\left|\mathbf{s}_{i}\right|=0 \quad \text { if } \quad(i, k) \in Q_{\tilde{B}}
$$

This condition is equivalent to

$$
\left|\overline{\mathbf{s}}_{j}\right|=0 \quad \text { if } \quad(k, j) \in Q_{B} \quad \text { and } \quad\left|\mathbf{s}_{i}\right|=0 \quad \text { if } \quad(i, k) \in Q_{B},
$$


because $\mathbf{s}_{i}=()$ for $i>n$ by convention. Such an $\mathbf{s}$ can be constructed as follows: since the initial seed is acyclic, $Q_{B}$ has no oriented cycles, therefore it determines a partial order where $i \prec j$ if there is a (directed) path from $i$ to $j$ in $Q_{B}$. Define $\mathbf{s}_{l} \in\{0,1\}^{\left[a_{l}\right]+}$ for $l=1, \ldots, n$ as

$$
\mathbf{s}_{l}= \begin{cases}(1, \ldots, 1), & \text { if } k \prec l, \\ (0, \ldots, 0), & \text { otherwise }\end{cases}
$$

Note that the construction of $\mathbf{s}$ depends on $k$. To check that $\mathbf{s}$ is in $S_{\mathrm{gcc}}$, we need to show that $\mathbf{s}_{i} \cdot \overline{\mathbf{s}}_{j}=0$ for every $(i, j) \in Q_{B}$. This can be proved by contradiction as follows. Assume $\mathbf{s}_{i} \cdot \overline{\mathbf{s}}_{j} \neq 0$ for some $(i, j) \in Q_{B}$. Then there exists $r \leq \min \left(\left[a_{i}\right]_{+},\left[a_{j}\right]_{+}\right)$such that $s_{i, r}=1, s_{j, r}=0$. By our choice of $\mathbf{s}$, we have $k \prec i, k \nprec j$, therefore $i \nprec j$. This contradicts with the assumption that $(i, j) \in Q_{B}$.

\section{Non-acyclic rank 3 cluster algebras}

In this section, we consider non-acyclic skew-symmetric rank 3 cluster algebras of geometric type.

\subsection{Definition and properties of $\tau$}

Let $m \geq 3$ be an integer, $\tilde{B}=\left(b_{i j}\right)$ be an $m \times 3$ matrix whose principal part $B$ is skew-symmetric and non-acyclic (i.e., $b_{12}, b_{23}, b_{31}$ are of the same sign).

Define the map

$$
\tau(B):=\left(\left|b_{23}\right|,\left|b_{31}\right|,\left|b_{12}\right|\right) \in \mathbb{Z}_{\geq 0}^{3} .
$$

Definition 4.1. Let $(x, y, z) \in \mathbb{R}^{3}$. We define a partial ordering " $\leq$ " on $\mathbb{R}^{3}$ by $(x, y, z) \leq$ $\left(x^{\prime}, y^{\prime}, z^{\prime}\right)$ if and only if $x \leq x^{\prime}, y \leq y^{\prime}$, and $z \leq z^{\prime}$.

Define three involutary functions $\mu_{1}, \mu_{2}, \mu_{3}: \mathbb{R}^{3} \rightarrow \mathbb{R}^{3}$ as follows

$$
\begin{aligned}
& \mu_{1}:(x, y, z) \mapsto(x, x z-y, z), \quad \mu_{2}:(x, y, z) \mapsto(x, y, x y-z), \\
& \mu_{3}:(x, y, z) \mapsto(y z-x, y, z) .
\end{aligned}
$$

Let $\Gamma$ be the group generated by $\mu_{1}, \mu_{2}$, and $\mu_{3}$. It follows that the $\Gamma$-orbit of $\tau(B)$ is identical to the set $\left\{\tau\left(B^{\prime}\right): B^{\prime}\right.$ is in the mutation class of $\left.B\right\}$.

Consider the following two situations.

(M1) $(a, b, c) \leq \mu_{i}(a, b, c)$ for all $i=1,2,3$.

(M2) $(a, b, c) \leq \mu_{i}(a, b, c)$ for precisely two indices $i$.

The following statement follows from [2, Theorem 1.2, Lemma 2.1] for a coefficient free cluster algebra. The result holds more generally for a cluster algebra of geometric type since the coefficients do not affect whether or not the seed is acyclic.

Theorem 4.2. Let $B$ be as above, $(a, b, c)=\tau(B)$. Then the following are equivalent:

(i) $\mathcal{A}(\mathbf{x}, \mathbf{y}, B)$ is non-acyclic.

(ii) $a, b, c \geq 2$ and $a b c+4 \geq a^{2}+b^{2}+c^{2}$.

(iii) $a, b, c \geq 2$ and there exists a unique triple in the $\Gamma$-orbit of $\tau(B)$ that satisfies (M1).

Moreover, under these conditions, triples in the $\Gamma$-orbit of $\tau(B)$ not satisfying (M1) must satisfy (M2). 
Assume $\mathcal{A}(\mathbf{x}, \mathbf{y}, B)$ is non-acyclic. We call the unique triple in Theorem 4.2(iii) the root of the $\Gamma$-orbit of $\tau(B)$.

Lemma 4.3. Let $B$ be as above, $(a, b, c)=\tau(B)$.

(i) The root $(a, b, c)$ of the $\Gamma$-orbit of $\tau(B)$ is the minimum of the $\Gamma$-orbit, i.e., $(a, b, c) \leq$ $\left(a^{\prime}, b^{\prime}, c^{\prime}\right)$ for any $\left(a^{\prime}, b^{\prime}, c^{\prime}\right)$ in the $\Gamma$-orbit.

(ii) If $\mu_{k}(a, b, c)=(a, b, c)$ for any $k$, then $a=b=c=2$ and $(2,2,2)$ is the unique triple in the $\Gamma$-orbit of $\tau(B)$. Therefore $(2,2,2)$ must also be the root.

(iii) Assume that $\tau(B)$ is the root of the $\Gamma$-orbit of $\tau(B), i_{1}, \ldots, i_{l} \in\{1,2,3\}, i_{s} \neq i_{s+1}$ for $1 \leq s \leq l-1$. Let $B_{0}=B, B_{j}=\mu_{i_{j}}\left(B_{j-1}\right)$ for $j=1, \ldots, l$. Then

$$
\tau\left(B_{0}\right) \leq \tau\left(B_{1}\right) \leq \cdots \leq \tau\left(B_{l}\right)
$$

Proof. (i) If $\tau\left(B^{\prime}\right)$ is the minimal triple in the $\Gamma$-orbit of $\tau(B)$ it satisfies (M1), so by Theorem 4.2(iii) the root is the unique triple satisfying (M1) and $B^{\prime}$ must be the root. For (ii): $a=b=c=2$ follows easily from Theorem 4.2(ii), and the uniqueness claim follows from the definition of $\mu_{k}$. (iii) is obvious if $\tau(B)=(2,2,2)$. If not, assume (iii) is false, then there are $1 \leq j<j^{\prime} \leq l$ such that

$$
\tau\left(B_{j}\right)<\tau\left(B_{j+1}\right)=\tau\left(B_{j+2}\right)=\cdots=\tau\left(B_{j^{\prime}}\right)>\tau\left(B_{j^{\prime}+1}\right) .
$$

By (ii) it must be that $j+1=j^{\prime}$ so that $\tau\left(B_{j}\right)<\tau\left(B_{j+1}\right)>\tau\left(B_{j+2}\right)$, but $\tau\left(B_{j+1}\right)$ does not satisfy either (M1) or (M2). This is impossible by Theorem 4.2(iii).

\subsection{Grading on $\mathcal{A}$}

We now adapt the grading introduced in [6, Definition 3.1] to the geometric type.

Definition 4.4. A graded seed is a quadruple $(\mathbf{x}, \mathbf{y}, B, G)$ such that

(i) $(\mathbf{x}, \mathbf{y}, B)$ is a seed of rank $n$, and

(ii) $G=\left[g_{1}, \ldots, g_{n}\right]^{T} \in \mathbb{Z}^{n}$ is an integer column vector such that $B G=0$.

Set $\operatorname{deg}_{G}\left(x_{i}\right)=g_{i}$ and $\operatorname{deg}\left(x_{i}^{-1}\right)=-g_{i}$ for $i \leq n$, and set $\operatorname{deg}\left(y_{j}\right)=0$ for all $j$. Extend the grading additively to Laurent monomials hence to the cluster algebra $\mathcal{A}(\mathbf{x}, \mathbf{y}, B)$. In [6] it is proved that under this grading every exchange relation is homogeneous and thus the grading is compatible with mutation.

Theorem 4.5 ([6, Corollary 3.4]). The cluster algebra $\mathcal{A}(\mathbf{x}, \mathbf{y}, B)$ under the above grading is $a \mathbb{Z}$-graded algebra.

The following two propositions come from the work in [15] to show that rank three non-acyclic cluster algebras have no maximal green sequences.

Theorem 4.6 ([15, Proposition 2.2]). Suppose that $B$ is a $3 \times 3$ skew-symmetric non-acyclic matrix. Then the (column) vector $G=\tau(B)^{T}$ satisfies $B G=0$.

Lemma 4.7. For any graded seed $\left(\mathbf{x}^{\prime}, B^{\prime}, G^{\prime}\right)$ in the mutation class of our initial graded seed, we have $G^{\prime}=\tau\left(B^{\prime}\right)^{T}$. 
Proof. This result follows from [15, Lemma 2.3], but its proof is short, so we reproduce it here. We use induction on mutations. Suppose that $G^{\prime}=\tau\left(B^{\prime}\right)^{T}$ for a given graded seed $\left(\mathbf{x}^{\prime}, B^{\prime}, G^{\prime}\right)$. Let $\left(\mathbf{x}^{\prime \prime}, B^{\prime \prime}, G^{\prime \prime}\right)$ be the graded seed obtained by taking mutation $\mu_{1}$ from $\left(\mathbf{x}^{\prime}, B^{\prime}, G^{\prime}\right)$. Then $x_{1}^{\prime \prime}=\left(\left(x_{2}^{\prime}\right)^{\left|b_{12}^{\prime}\right|}+\left(x_{3}^{\prime}\right)^{\left|b_{13}^{\prime}\right|}\right) / x_{1}^{\prime}$, so its degree is $g_{2}^{\prime}\left|b_{12}^{\prime}\right|-g_{1}^{\prime}$. By induction we have

$$
g_{2}^{\prime}\left|b_{12}^{\prime}\right|-g_{1}^{\prime}=\left|b_{31}^{\prime}\right|\left|b_{12}^{\prime}\right|-\left|b_{23}^{\prime}\right|=\left|b_{31}^{\prime} b_{12}^{\prime}-b_{23}^{\prime}\right|=\left|b_{23}^{\prime \prime}\right|,
$$

so we get $G^{\prime \prime}=\tau\left(B^{\prime \prime}\right)^{T}$. The cases of $\mu_{2}$ and $\mu_{3}$ are similar.

In the rest of Section 4 we assume that $B$ is a $3 \times 3$ skew-symmetric non-acyclic matrix and $G=\tau(B)^{T}$. In other words, if $\tau(B)=(b, c, a)$ then $\operatorname{deg}\left(x_{1}\right)=b, \operatorname{deg}\left(x_{2}\right)=c, \operatorname{deg}\left(x_{3}\right)=a$ for any seed $(\mathbf{x}, \mathbf{y}, B)$. If we look at the quiver associated to our exchange matrix we see that the degree of the cluster variable $x_{i}$ is the number of arrows between the other two mutable vertices in $Q_{B}$. Furthermore, this is a canonical grading for non-acyclic rank three cluster algebras since regardless of your choice of initial seed the grading imposed on $\mathcal{A}$ is the same.

\subsection{Construction of an element in $\mathcal{U} \backslash \mathcal{A}$}

Here we shall prove the following main theorem of the paper.

We give $\mathcal{A}$ the $\mathbb{Z}$-grading as in Section 4.2. By Theorem 4.2, we can assume that the initial seed

$$
\Sigma=\left(\left\{x_{1}, x_{2}, x_{3}\right\}, \mathbf{y}, B, G\right), \quad \text { where } \quad B=\left[\begin{array}{ccc}
0 & a & -c \\
-a & 0 & b \\
c & -b & 0
\end{array}\right], \quad G=\left[\begin{array}{l}
b \\
c \\
a
\end{array}\right]
$$

satisfies $a, b, c \geq 2$ and (M1). Then $\operatorname{deg} x_{1}=b, \operatorname{deg} x_{2}=c, \operatorname{deg} x_{3}=a$, and we let $x_{4}, \ldots, x_{m}$ be of degree 0 . Furthermore, by permuting the indices if necessary, we assume $a \geq b \geq c$.

Define six degree-0 elements as follows

$$
\alpha_{i}^{ \pm}:=\prod_{j=4}^{m} x_{j}^{\left[ \pm b_{j i}\right]_{+}}, \quad i=1,2,3 \text {. }
$$

Looking at the seeds neighboring $\Sigma$ we obtain three new cluster variables. Namely,

$$
z_{1}=\frac{\alpha_{1}^{-} x_{2}^{a}+\alpha_{1}^{+} x_{3}^{c}}{x_{1}}, \quad z_{2}=\frac{\alpha_{2}^{+} x_{1}^{a}+\alpha_{2}^{-} x_{3}^{b}}{x_{2}}, \quad z_{3}=\frac{\alpha_{3}^{-} x_{1}^{c}+\alpha_{3}^{+} x_{2}^{b}}{x_{3}}
$$

which have degree $a c-b, a b-c$, and $b c-a$, respectively.

We also use the following theorem, whose proof in [11] is for coefficient free cluster algebras but clearly applies to cluster algebras of geometric type.

Theorem 4.8 ([11, Proposition 6.1.2]). If $\mathcal{A}$ is a rank three skew-symmetric non-acyclic cluster algebra, then $\mathcal{A}$ is totally coprime.

Now we consider a special element in $\mathbb{Q}\left(x_{1}, x_{2}, x_{3}\right)$ :

$$
Y:=\tilde{x}[(1,0,1)] / x_{2}^{b}=\frac{\alpha_{1}^{-} \alpha_{3}^{-} x_{1}^{c} x_{2}^{a-b}+\alpha_{1}^{-} \alpha_{3}^{+} x_{2}^{a}+\alpha_{1}^{+} \alpha_{3}^{+} x_{3}^{c}}{x_{1} x_{3}} .
$$

We shall prove Theorem 1.3 by showing that the element constructed above is in $\mathcal{U} \backslash \mathcal{A}$. 
Proof of Theorem 1.3. We first show that $Y \in \mathcal{U}$. Note that $\mathcal{A}$ is a rank 3 cluster algebra so it is totally coprime by Theorem 4.8. It then suffices to show that $Y \in \mathcal{U}_{\mathbf{x}}$ by Theorem 2.4. Clearly $Y \in \mathbb{Z} \mathbb{P}\left[x_{1}^{ \pm 1}, x_{2}^{ \pm 1}, x_{3}^{ \pm 1}\right]$, where $\mathbb{Z P}=\mathbb{Z}\left[x_{4}^{ \pm 1}, \ldots, x_{m}^{ \pm 1}\right]$. Also,

$$
\begin{aligned}
& Y=\frac{\alpha_{3}^{+} z_{1}^{c}+\alpha_{1}^{-} \alpha_{3}^{-}\left(\alpha_{1}^{-} x_{2}^{a}+\alpha_{1}^{+} x_{3}^{c}\right)^{c-1} x_{2}^{a-b}}{z_{1}^{c-1} x_{3}} \in \mathbb{Z} \mathbb{P}\left[z_{1}^{ \pm 1}, x_{2}^{ \pm 1}, x_{3}^{ \pm 1}\right], \\
& Y=\frac{\alpha_{1}^{-} \alpha_{3}^{-} x_{1}^{c}\left(\alpha_{2}^{+} x_{1}^{a}+\alpha_{2}^{-} x_{3}^{b}\right)^{a-b} z_{2}^{b}+\alpha_{1}^{+} \alpha_{3}^{+} z_{2}^{a} x_{3}^{c}+\alpha_{1}^{-} \alpha_{3}^{+}\left(\alpha_{2}^{+} x_{1}^{a}+\alpha_{2}^{-} x_{3}^{b}\right)^{a}}{x_{1} z_{2}^{a} x_{3}} \in \mathbb{Z} \mathbb{P}\left[x_{1}^{ \pm 1}, z_{2}^{ \pm 1}, x_{3}^{ \pm 1}\right], \\
& Y=\frac{\alpha_{1}^{-} x_{2}^{a-b} z_{3}^{c}+\alpha_{1}^{+} \alpha_{3}^{+}\left(\alpha_{3}^{-} x_{1}^{c}+\alpha_{3}^{+} x_{2}^{b}\right)^{c-1}}{x_{1} z_{3}^{c-1}} \in \mathbb{Z} \mathbb{P}\left[x_{1}^{ \pm 1}, x_{2}^{ \pm 1}, z_{3}^{ \pm 1}\right] .
\end{aligned}
$$

Therefore we conclude that $Y \in \mathcal{U}_{\mathbf{x}}$.

Next, we show that $Y \notin \mathcal{A}$. With respect to our grading of $\mathcal{A}, Y$ is homogeneous of degree $a c-b-a$.

Combining Lemmas 4.3 and 4.7 we have already shown that the degree of cluster variables is non-decreasing as we mutate away from our initial seed. We use this fact to explicitly prove that all cluster variables, except possibly $x_{1}, x_{2}, x_{3}, z_{3}$, have degree strictly larger than $a c-b-a$. Indeed, let $x$ be a cluster variable such that $x \neq x_{1}, x_{2}, x_{3}, z_{3}$. Then $x$ can be written as

$$
x=\mu_{i_{l}} \cdots \mu_{i_{2}} \mu_{i_{1}}\left(x_{k}\right), \quad i_{1}, \ldots, i_{l}, k \in\{1,2,3\}, \quad \text { and } \quad i_{s} \neq i_{s+1} \quad \text { for } 1 \leq s \leq l-1 .
$$

Let $B_{0}=B, B_{j}=\mu_{i_{j}}\left(B_{j-1}\right)$ for $j=1, \ldots, l$. Let $\tau\left(B_{j}\right)=\left(b_{j}, c_{j}, a_{j}\right)$ for $j=0, \ldots, l$. By Lemma 4.3, we have that

$$
(b, c, a)=\left(b_{0}, c_{0}, a_{0}\right) \leq\left(b_{1}, c_{1}, a_{1}\right) \leq \cdots \leq\left(b_{l}, c_{l}, a_{l}\right) .
$$

We prove the claim in the following five cases.

Case $k=1$. We let $r>0$ be the smallest integer such that $i_{r}=k$ (which exists since $\left.x \neq x_{1}, x_{2}, x_{3}\right)$. It suffices to prove that the degree of $w=\mu_{i_{r}} \cdots \mu_{i_{2}} \mu_{i_{1}}\left(x_{k}\right)$ is larger than $a c-b-a$. Indeed,

$$
\operatorname{deg} w=b_{r}=a_{r-1} c_{r-1}-b_{r-1}=a_{r-1} c_{r-1}-b \geq a c-b>a c-b-a .
$$

Case $k=2$. The above proof still works:

$$
\operatorname{deg} w=c_{r}=a_{r-1} b_{r-1}-c_{r-1}=a_{r-1} b_{r-1}-c \geq a b-c>a c-b-a .
$$

Case $k=3, i_{1}=1$. We let $r>0$ be the smallest integer such that $i_{r}=k$. Then $\left(b_{1}, c_{1}, a_{1}\right)=$ $(a c-b, c, a)$, and

$$
\operatorname{deg} w=a_{r}=b_{r-1} c_{r-1}-a_{r-1}=b_{r-1} c_{r-1}-a \geq b_{1} c_{1}-a=(a c-b) c-a>a c-b-a .
$$

Case $k=3, i_{1}=2$. Similar to the above case, $\left(b_{1}, c_{1}, a_{1}\right)=(b, a b-c, a)$,

$$
\operatorname{deg} w=a_{r} \geq b_{1} c_{1}-a=b(a b-c)-a \geq b^{2}(a-1)-a>c(a-1)-a \geq a c-b-a .
$$

The second and fourth inequality follow from our assumption that $b \geq c$.

Case $k=3, i_{1}=3$. Then $\left(b_{1}, c_{1}, a_{1}\right)=(b, c, b c-a), i_{2} \neq 3$. We let $r \geq 3$ be the smallest integer such that $i_{r}=k$ (which exists since $x \neq z_{3}$ ). It suffices to prove that the degree of $w=\mu_{i_{r}} \cdots \mu_{i_{2}} \mu_{i_{1}}\left(x_{k}\right)$ is larger than $a c-b-a$.

If $i_{2}=1$, then $\left(b_{2}, c_{2}, a_{2}\right)=(c(b c-a)-b, c, b c-a)$,

$$
\begin{aligned}
\operatorname{deg} w & =a_{r}=b_{r-1} c_{r-1}-(b c-a) \geq b_{2} c_{2}-(b c-a)=(c(b c-a)-b) c-(b c-a) \\
& =\left(c^{2}-2\right)(b c-a)-a \geq\left(c^{2}-2\right) a-a=a\left(c^{2}-3\right) \geq a(c-1)>a c-b-a .
\end{aligned}
$$




$$
\begin{aligned}
& \text { If } i_{2}=2 \text {, then }\left(b_{2}, c_{2}, a_{2}\right)=(b,(b c-a) b-c, b c-a), \\
& \qquad \operatorname{deg} w \geq b((b c-a) b-c)-(b c-a) \geq(c(b c-a)-b) c-(b c-a)>a c-b-a .
\end{aligned}
$$

The second inequality above follows from our assumption that $b \geq c$. One application gives the inequality $(b c-a) b-c \geq c(b c-a)-b$ and a second application replaces a factor of $b$ in the first term with a factor of $c$. The last inequality follows from our computation in the previous case.

This completes the proof of the claim that all cluster variables, except possibly $x_{1}, x_{2}, x_{3}, z_{3}$, have degree strictly larger than $a c-b-a$.

Therefore it is sufficient to check that $Y$ cannot be written as a linear combination of products of the cluster variables $x_{1}, x_{2}, x_{3}$, and $z_{3}$ since all other cluster variable have larger degree than $Y$. This is clear as the numerator of $Y$ is irreducible and none of these cluster variables have a factor of $x_{1}$ in the denominator.

\section{$5 \quad$ Dyck path formula}

In this section, we give the Dyck path construction of $\tilde{x}[\mathbf{a}]$ that is equivalent to Section 3 (under a mild condition that there is no isolated vertex in the digraph $Q_{\tilde{B}}$ ). It is in fact our original definition of $\tilde{x}[\mathbf{a}]$, and appears naturally in the attempt of generalizing greedy bases for cluster algebras of rank 2 in [9] and the construction of bases of type $A$ cluster algebras in [1] to more general cases.

Let $\left(a_{1}, a_{2}\right)$ be a pair of nonnegative integers. Let $c=\min \left(a_{1}, a_{2}\right)$. The maximal Dyck path of type $a_{1} \times a_{2}$, denoted by $\mathcal{D}=\mathcal{D}^{a_{1} \times a_{2}}$, is a lattice path from $(0,0)$ to $\left(a_{1}, a_{2}\right)$ that is as close as possible to the diagonal joining $(0,0)$ and $\left(a_{1}, a_{2}\right)$, but never goes above it. A corner is a subpath consisting of a horizontal edge followed by a vertical edge.

Definition 5.1. Let $\mathcal{D}_{1}$ (resp. $\mathcal{D}_{2}$ ) be the set of horizontal (resp. vertical) edges of a maximal Dyck path $\mathcal{D}=\mathcal{D}^{a_{1} \times a_{2}}$. We label $\mathcal{D}$ with the corner-first index in the following sense:

(a) edges in $\mathcal{D}_{1}$ are indexed as $u_{1}, \ldots, u_{a_{1}}$ such that $u_{i}$ is the horizontal edge of the $i$-th corner for $i \in[1, c]$ and $u_{c+i}$ is the $i$-th of the remaining horizontal ones for $i \in\left[1, a_{1}-c\right]$,

(b) edges in $\mathcal{D}_{2}$ are indexed as $v_{1}, \ldots, v_{a_{2}}$ such that $v_{i}$ is the vertical edge of the $i$-th corner for $i \in[1, c]$ and $v_{c+i}$ is the $i$-th of the remaining vertical ones for $i \in\left[1, a_{2}-c\right]$.

Here we count corners from bottom left to top right, count vertical edges from bottom to top, and count horizontal edges from left to right.)

Definition 5.2. Let $S_{1} \subseteq \mathcal{D}_{1}$ and $S_{2} \subseteq \mathcal{D}_{2}$. We say that $S_{1}$ and $S_{2}$ are locally compatible with respect to $\mathcal{D}$ if and only if no horizontal edge in $S_{1}$ is the immediate predecessor of any vertical edge in $S_{2}$ on $\mathcal{D}$. In other words, the subpath $S_{1} \cup S_{2}$ contains no corners.

Remark 5.3. Notice that we have: $\left|\mathcal{P}\left(\mathcal{D}_{1}\right) \times \mathcal{P}\left(\mathcal{D}_{2}\right)\right|$ possible pairs for $\left(S_{1}, S_{2}\right)$, where $\mathcal{P}\left(\mathcal{D}_{1}\right)$ denotes the power set of $\mathcal{D}_{1}$ and $\mathcal{P}\left(\mathcal{D}_{2}\right)$ denotes the power set of $\mathcal{D}_{2}$. Further, when either $S_{1}$ or $S_{2}=\varnothing$, any arbitrary choice of the other will yield local compatibility by our above definition.

Definition 5.4. Let $\mathbf{a}=\left(a_{i}\right) \in \mathbb{Z}^{n}$. By convention we assume $a_{i}=0$ for $i>n$. For each pair $(i, j) \in Q_{\tilde{B}}$ (defined at the beginning of Section 3), denote $\mathcal{D}^{\left[a_{i}\right]_{+} \times\left[a_{j}\right]_{+}}$by $\mathcal{D}^{(i, j)}$. We label $\mathcal{D}^{(i, j)}$ with the corner-first index (Definition 5.1), whose horizontal edges are denoted $u_{1}^{(i, j)}, \ldots, u_{\left[a_{i}\right]_{+}}^{(i, j)}$ and vertical edges are denoted by $v_{1}^{(i, j)}, \ldots, v_{\left[a_{j}\right]_{+}}^{(i, j)}$. We say that the collection

$$
\left\{S_{\ell}^{(i, j)} \subseteq \mathcal{D}_{\ell}^{(i, j)} \mid(i, j) \in Q_{\tilde{B}}, \ell \in\{1,2\}\right\}
$$

is globally compatible if and only if 
(i) $S_{1}^{(i, j)}$ and $S_{2}^{(i, j)}$ are locally compatible with respect to $\mathcal{D}^{(i, j)}$ for all $(i, j) \in Q_{\tilde{B}}$;

(ii) if $(i, j)$ and $(j, k)$ are in $Q_{\tilde{B}}$, then $v_{r}^{(i, j)} \in S_{2}^{(i, j)} \Longleftrightarrow u_{r}^{(j, k)} \notin S_{1}^{(j, k)}$ for all $r \in\left[1,\left[a_{j}\right]_{+}\right]$;

(iii) if $(j, k)$ and $(j, i)$ are in $Q_{\tilde{B}}$, then $u_{r}^{(j, k)} \in S_{1}^{(j, k)} \Longleftrightarrow u_{r}^{(j, i)} \in S_{1}^{(j, i)}$ for all $r \in\left[1,\left[a_{j}\right]_{+}\right]$;

(iv) if $(i, j)$ and $(k, j)$ are in $Q_{\tilde{B}}$, then $v_{r}^{(i, j)} \in S_{2}^{(i, j)} \Longleftrightarrow v_{r}^{(k, j)} \in S_{2}^{(k, j)}$ for all $r \in\left[1,\left[a_{j}\right]_{+}\right]$.

We say that the collection is quasi-compatible if it satisfies (ii), (iii), and (iv). So a globally compatible collection is also a quasi-compatible collection. For an illustration of the conditions, see Fig. 1.

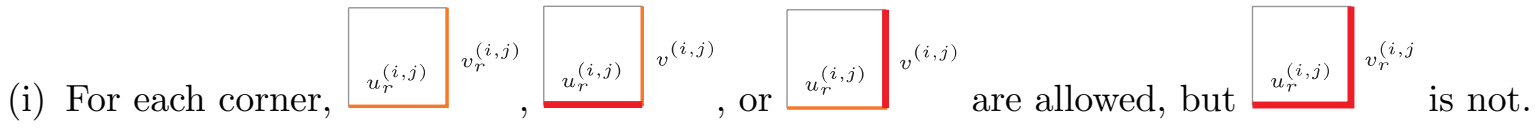

(ii)

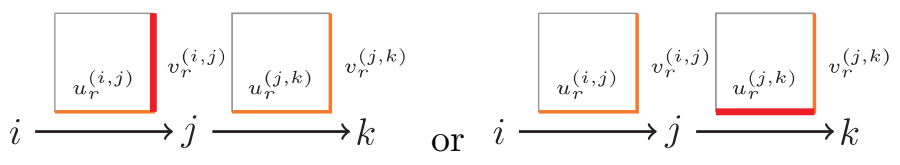

(iii)

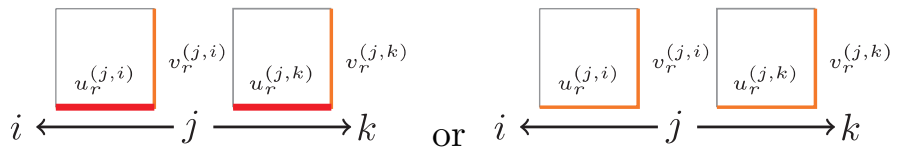

(iv)

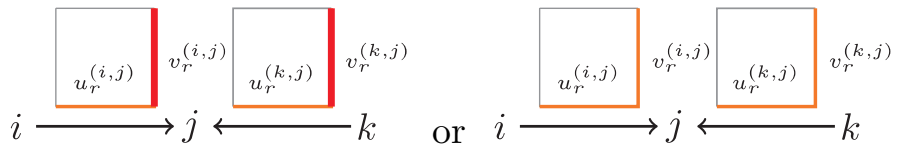

Figure 1. Pictorial descriptions for Definition 5.4(i)-(iv).

Proposition 5.5. Same notation as in Definition 5.4. Assume that there is no isolated vertex in the digraph $Q_{\tilde{B}}$. Then

$$
\tilde{x}[\mathbf{a}]=\left(\prod_{l=1}^{n} x_{l}^{-a_{l}}\right) \sum\left(\prod_{(i, j) \in Q_{\tilde{B}}} x_{i}^{b_{i j}\left|S_{2}^{(i, j)}\right|} x_{j}^{-b_{j i}\left|S_{1}^{(i, j)}\right|}\right),
$$

where the sum runs over all globally compatible collections (abbreviated GCCs). A similar formula holds for $z[\mathbf{a}]$ if the sum runs over all quasi-compatible collections.

Proof. We define a mapping that sends a globally compatible collection

$$
\left\{S_{\ell}^{(i, j)} \subseteq \mathcal{D}_{\ell}^{(i, j)} \mid(i, j) \in Q_{\tilde{B}}, \ell \in\{1,2\}\right\},
$$

to a sequence of sequences $\mathbf{s}=\left(\mathbf{s}_{1}, \ldots, \mathbf{s}_{n}\right)$ as follows. For $1 \leq j \leq n$ and $1 \leq r \leq\left[a_{j}\right]_{+}$, the $r$-th number $s_{j, r}$ of the sequence $\mathbf{s}_{j}=\left(s_{j, 1}, s_{j, 2}, \ldots, s_{j,\left[a_{j}\right]_{+}}\right) \in\{0,1\}^{\left[a_{j}\right]_{+}}$is determined by

(i) if $(i, j) \in Q_{\tilde{B}}$, then $v_{r}^{(i, j)} \in S_{2}^{(i, j)}$ if and only if $s_{j, r}=0$;

(ii) if $(j, k) \in Q_{\tilde{B}}$, then $u_{r}^{(j, k)} \in S_{1}^{(j, k)}$ if and only if $s_{j, r}=1$.

Since we assume that $Q_{\tilde{B}}$ has no isolated vertices and the collection $\left\{S_{\ell}^{(i, j)}\right\}$ are globally compatible, all $s_{j, r}$ are well-defined. Noting that the number of corners in $\mathcal{D}^{(i, j)}$ is $\min \left(\left[a_{i}\right]_{+},\left[a_{j}\right]_{+}\right)$ and that we use the corner-first index, it is easy to conclude that $\mathbf{s}$ is indeed in $S_{\text {gcc }}$, and that the mapping is bijective and the exponents that appear in $\tilde{x}[\mathbf{a}]$ work out correctly.

The formula for $z[a]$ is proved similarly. 
Remark 5.6. Note that the local compatibility condition in Definition 5.4(i) is weaker than the compatibility condition for greedy elements given in [9]. Even for rank 2 cluster algebras, not all cluster variables are of the form $\tilde{x}[\mathbf{a}]$. As an example,

$$
B=\left[\begin{array}{cc}
0 & 2 \\
-2 & 0
\end{array}\right]
$$

Then by Lemma 3.3(i),

$$
\tilde{x}[(1,2)]=\tilde{x}[(1,1)] \tilde{x}[(0,1)]=\left(\frac{1+x_{1}^{2}+x_{2}^{2}}{x_{1} x_{2}}\right)\left(\frac{1+x_{1}^{2}}{x_{2}}\right)=\frac{1+2 x_{1}^{2}+x_{1}^{4}+x_{2}^{2}+x_{1}^{2} x_{2}^{2}}{x_{1} x_{2}^{2}},
$$

which is not equal to the following cluster variable (which is a greedy element)

$$
x[(1,2)]=\frac{1+2 x_{1}^{2}+x_{1}^{4}+x_{2}^{2}}{x_{1} x_{2}^{2}} .
$$

Other $\tilde{x}[\mathbf{a}]$ are not equal to this cluster variable either, since their denominators do not match. It is illustrating to also compare with the standard monomial basis element

$$
z[(1,2)]=\left(\frac{1+x_{2}^{2}}{x_{1}}\right)\left(\frac{1+x_{1}^{2}}{x_{2}}\right)^{2}=\frac{1+2 x_{1}^{2}+x_{1}^{4}+x_{2}^{2}+2 x_{1}^{2} x_{2}^{2}+x_{1}^{4} x_{2}^{2}}{x_{1} x_{2}^{2}} .
$$

Similar as the standard monomial basis, $\{\tilde{x}[\mathbf{a}]\}$ also depends on the initial cluster in general.

For interested readers, we explain the above example using pictures: in Fig. 2, all 8 possible collections of edges in $\mathcal{D}^{1 \times 2}$ together with their contributions to the numerator of $z[(1,2)]$ are listed; among them, the first 6 are GCCs and contribute to the numerator of $\tilde{x}[(1,2)]$, and the first 5 are compatible pairs in the definition of greedy elements and contribute to the numerator of $x[(1,2)]$.

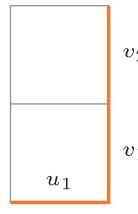

1
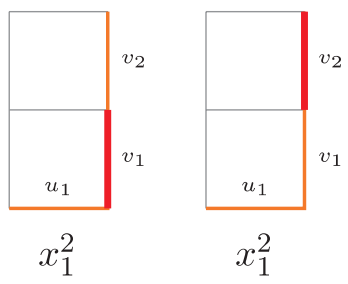
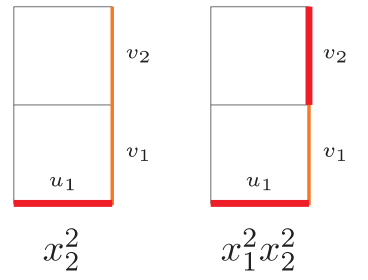
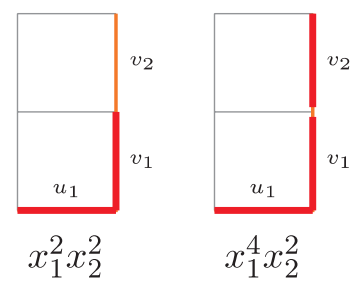

Figure 2. Qausi-compatible collections for $\mathbf{a}=(1,2)$.

\section{Proof of Theorem 1.2}

In this section, we give two proofs of the fact that $\{\tilde{x}[\mathbf{a}]\}_{\mathbf{a} \in \mathbb{Z}^{n}}$ constructed from an acyclic seed (i.e., $Q_{B}$ is acyclic) form a basis of an acyclic cluster algebra $\mathcal{A}$ of geometric type, using different approaches. The main idea behind both proofs is to compare $\{\tilde{x}[\mathbf{a}]\}_{\mathbf{a} \in \mathbb{Z}^{n}}$ with the standard monomial basis $\{z[\mathbf{a}]\}_{\mathbf{a} \in \mathbb{Z}^{n}}$ which is known to have the desired property. The first approach is to consider certain orders on Laurent monomials and use the fact that $\tilde{x}[\mathbf{a}]$ and $z[\mathbf{a}]$ have the same lowest Laurent monomial to draw the conclusion. The second approach is to use the multiplicative property of $\tilde{x}[\mathbf{a}]$ and $z[\mathbf{a}]$ and the combinatorial descriptions of these elements. 


\subsection{The first proof}

Note that $Q_{B}$ is acyclic by assumption. If $Q_{B}$ has only one vertex, then $\tilde{x}[\mathbf{a}]=z[\mathbf{a}]$ and we are done. If $Q_{B}$ is not weakly connected (recall that a digraph is weakly connected if replacing all of its directed edges with undirected edges produces a connected undirected graph), we can factorize $\tilde{x}[\mathbf{a}]$ (resp. $z[\mathbf{a}]$ ) into a product of those with smaller ranks, each corresponds to a connected component (see Lemma 3.3(ii)). Thus we can reduce to the situation that $Q_{B}$ is weakly connected and $n \geq 2$. Relabeling vertices $1, \ldots, n$ if necessary, we may assume that

$$
\text { if } i \rightarrow j \text { is in } Q_{B} \text {, then } i<j \text {. }
$$

Let $\left\{e_{i}\right\}$ be the standard basis of $\mathbb{Z}^{n}$, and $\left(r_{1}, \ldots, r_{n}\right)$ is a permutation of $\{1, \ldots, n\}$. We say that a map $f: \mathbb{Z}^{n} \rightarrow \mathbb{Z}^{n}$ is coordinate-wise $\mathbb{Z}_{\geq 0}$-linear if

$$
f\left(\sum_{i} m_{i} \epsilon_{i} e_{i}\right)=\sum_{i} m_{i} f\left(\epsilon_{i} e_{i}\right), \quad \text { for all } m_{i} \in \mathbb{Z}_{\geq 0}, \quad \epsilon_{i} \in\{1,-1\} .
$$

We call such a map triangularizable if there is an order on the set $\{1, \ldots, n\}$ as $\left\{r_{1}, \ldots, r_{n}\right\}$ such that

$$
f\left(\epsilon e_{r_{i}}\right)+\epsilon e_{r_{i}} \in \mathbb{Z} e_{r_{i+1}} \oplus \cdots \oplus \mathbb{Z} e_{r_{n}}, \quad \forall 1 \leq i \leq n, \quad \epsilon \in\{1,-1\} .
$$

Lemma 6.1. Let $f$ be a triangularizable coordinate-wise $\mathbb{Z}_{\geq 0}$-linear map. Then $f$ is bijective.

Proof. Without loss of generality we assume $r_{i}=i$ for $i=1, \ldots, n$. We show that $f$ is invertible, i.e., for any $\sum c_{i} e_{i} \in \mathbb{Z}^{n}$, there exists a unique $\sum b_{i} e_{i} \in \mathbb{Z}^{n}$ such that

$$
f\left(\sum b_{i} e_{i}\right)=\sum c_{i} e_{i}
$$

We first show that $f$ is injective. Assume $\sum b_{i} e_{i}$ satisfies (6.1). Define a function

$$
s: \mathbb{Z} \rightarrow\{1,-1\}, \quad s(b)=\left\{\begin{aligned}
1, & \text { if } b \geq 0, \\
-1, & \text { otherwise }
\end{aligned}\right.
$$

Denote the usual inner product on $\mathbb{Z}^{n}$ where $\left\langle e_{i}, e_{j}\right\rangle=1$ if $i=j$, otherwise 0 . Then for each $1 \leq k \leq n$, we must have

$$
\begin{aligned}
c_{k} & =\left\langle\sum c_{i} e_{i}, e_{k}\right\rangle=\sum_{i=1}^{n}\left|b_{i}\right|\left\langle f\left(s\left(b_{i}\right) e_{i}\right), e_{k}\right\rangle \\
& =\left(\sum_{i=1}^{k-1}\left|b_{i}\right|\left\langle f\left(s\left(b_{i}\right) e_{i}\right), e_{k}\right\rangle\right)+\left|b_{k}\right|\left\langle f\left(s\left(b_{k}\right) e_{k}\right), e_{k}\right\rangle=\left(\sum_{i=1}^{k-1}\left|b_{i}\right|\left\langle f\left(s\left(b_{i}\right) e_{i}\right), e_{k}\right\rangle\right)-b_{k},
\end{aligned}
$$

where the third equality is because of the following: for $i>k$, the triangularizable property assures that $f\left( \pm e_{i}\right)$ would be written only using a linear combination of $e_{j}$ 's with $j>k$; since $\left\langle e_{j}, e_{k}\right\rangle=0$ for these $j$ 's, we have $\left\langle f\left( \pm e_{i}\right), e_{k}\right\rangle=0$. Therefore $b_{1}, \ldots, b_{n}$ are uniquely determined (in that order) by the equation

$$
b_{k}=\left(\sum_{i=1}^{k-1}\left|b_{i}\right|\left\langle f\left(s\left(b_{i}\right) e_{i}\right), e_{k}\right\rangle\right)-c_{k}
$$

implying that $f$ is injective. 
To show that $f$ is surjective, we construct $b_{1}, \ldots, b_{n}$, in that order, by (6.2). Then we claim that (6.1) holds. Indeed,

$$
\left\langle f\left(\sum b_{i} e_{i}\right), e_{k}\right\rangle=\sum_{i=1}^{n}\left|b_{i}\right|\left\langle f\left(s\left(b_{i}\right) e_{i}\right), e_{k}\right\rangle=\left(\sum_{i=1}^{k-1}\left|b_{i}\right|\left\langle f\left(s\left(b_{i}\right) e_{i}\right), e_{k}\right\rangle\right)-b_{k}=c_{k}
$$

for all $1 \leq k \leq n$, which implies (6.1).

Take the lexicographic order

$$
x_{1}>x_{2}>\cdots>x_{n}>1,
$$

that is, $\prod x_{i}^{a_{i}}<\prod x_{i}^{b_{i}}$ if the first pair of unequal exponents $a_{i}$ and $b_{i}$ satisfy $a_{i}<b_{i}$.

Lemma 6.2. The map $f: \mathbb{Z}^{n} \rightarrow \mathbb{Z}^{n}$ sending a to the exponent vector of the lowest Laurent monomial of $z[\mathbf{a}]$ is triangularizable coordinate-wise $\mathbb{Z}_{\geq 0}$-linear, so is injective.

Moreover, the coefficient of the lowest Laurent monomial of $z[\mathbf{a}]$ (which a priori is a Laurent polynomial in $\mathbb{Z} \mathbb{P})$ is a Laurent monomial in $\mathbb{Z P}$, i.e., invertible in $\mathbb{Z} \mathbb{P}$.

Proof. It follows from the definition of $z[\mathbf{a}]$ that $f$ is coordinate-wise $\mathbb{Z}_{\geq 0}$-linear. Next we show that $f$ is triangularizable. Take $1 \leq k \leq n$. By the definition of mutation,

$$
x_{k}^{\prime}=x_{k}^{-1}(\underbrace{\prod_{1 \leq i<k} x_{i}^{b_{i k}}}_{P_{1}} \underbrace{\prod_{i>n} x_{i}^{\left[b_{i k}\right]_{+}}}_{Q_{1}}+\underbrace{\prod_{k<i \leq n} x_{i}^{-b_{i k}}}_{P_{2}} \underbrace{\prod_{i>n} x_{i}^{\left[-b_{i k}\right]_{+}}}_{Q_{2}}),
$$

so its lowest monomial is

$$
\begin{cases}x_{k}^{-1} P_{2} Q_{2}, & \text { if } k \text { is not a source in } Q_{B}, \\ x_{k}^{-1} P_{1} Q_{1}=x_{k}^{-1} Q_{1}, & \text { if } k \text { is a source in } Q_{B} .\end{cases}
$$

So

$$
f\left(e_{k}\right)= \begin{cases}-e_{k}+\sum_{k<i \leq n}\left(-b_{i k}\right) e_{i}, & \text { if } k \text { is not a source in } Q_{B} \\ -e_{k}, & \text { if } k \text { is a source in } Q_{B}\end{cases}
$$

We also have the obvious equality $f\left(-e_{k}\right)=e_{k}$. Thus $f$ is triangularizable by taking the order $(1,2, \ldots, n)$. Therefore, $f$ is injective by Lemma 6.1 .

For the "moreover" statement, it suffices to note that $P_{1}$ and $P_{2}$ in the above definition of $x_{k}^{\prime}$ have different exponent vectors. This is because of the assumption that $n \geq 2$ and $Q_{B}$ is weakly connected, so it is impossible for $P_{1}=P_{2}=1$ to hold.

Lemma 6.3. For any $\mathbf{a} \in \mathbb{Z}^{n}$, the lowest Laurent monomials in $z[\mathbf{a}]$ and $\tilde{x}[\mathbf{a}]$ are equal. As a consequence, $\{\tilde{x}[\mathbf{a}]\} \mathbf{a} \in \mathbb{Z}^{n}$ is $\mathbb{Z} \mathbb{P}$-linearly independent.

Proof. First note that the consequence follows from Lemma 6.2, so for the rest we focus on the first statement. By the multiplicative property of $z[\mathbf{a}]$ and $\tilde{x}[\mathbf{a}]$ (Lemma 3.3), if suffices to consider the case when $\mathbf{a}=\left(a_{i}\right) \in\{0,1\}^{n}$. In the following, we use the notation from Lemma 3.5. Define

$$
J:=\left\{1 \leq k \leq n \mid a_{k}=1\right\}, \quad H:=\left\{1 \leq k \leq n \mid k \text { is a source in } Q_{B}\right\}
$$


Since $z[\mathbf{a}]=\prod_{k \in J} x_{k}^{\prime}$, its lowest Laurent monomial is

$$
\left(\prod_{l=1}^{n} x_{l}^{-a_{l}}\right)\left(\prod_{k \in J \backslash H}\left(\prod_{k<i \leq n} x_{i}^{-b_{i k}} \prod_{i>n} x_{i}^{\left[-b_{i k}\right]_{+}}\right)\right)\left(\prod_{k \in J \cap H} \prod_{i>n} x_{i}^{\left[b_{i k}\right]_{+}}\right) .
$$

Define $\mathbf{s}=\left(s_{1}, \ldots, s_{n}\right)$ by $s_{k}=1$ if $k \in J \backslash H$, otherwise take $s_{k}=0$. For $i \leq n$, the degree of $x_{i}$ in $(6.3)$ is

$$
-a_{i}+\sum_{k \in J \backslash H}\left[-b_{i k}\right]_{+}=-a_{i}+\sum_{k=1}^{n} s_{k}\left[-b_{i k}\right]_{+}=-a_{i}+\sum_{k=1}^{n}\left(s_{k}\left[-b_{i k}\right]_{+}+\left(a_{k}-s_{k}\right)\left[b_{i k}\right]_{+}\right),
$$

where the second equality is because $\left(a_{k}-s_{k}\right)\left[b_{i k}\right]_{+}=0$. Indeed, if $a_{k}-s_{k} \neq 0$, then $a_{k}=1$ and $s_{k}=0$, thus $k \in J \cap H$. Then $k \in H$ implies $b_{i k} \leq 0$, therefore $\left[-b_{i k}\right]_{+}=0$. For $i>n$, the degree of $x_{i}$ in (6.3) is

$$
\sum_{k \in J \backslash H}\left[-b_{i k}\right]_{+}+\sum_{k \in J \cap H}\left[b_{i k}\right]_{+}=\sum_{k=1}^{n} s_{k}\left[-b_{i k}\right]_{+}+\left(a_{k}-s_{k}\right)\left[b_{i k}\right]_{+} .
$$

So we can rewrite $(6.3)$ as

$$
\left(\prod_{i=1}^{n} x_{i}^{-a_{i}}\right) \prod_{i=1}^{m} x_{i}^{\sum_{j=1}^{n}\left(a_{j}-s_{j}\right)\left[b_{i j}\right]_{+}+s_{j}\left[-b_{i j}\right]_{+}} .
$$

It is easy to check that $\mathbf{s}$ is in $\mathbf{S}$. Comparing with (3.2) in Lemma 3.5 we draw the expected conclusion.

Lemma 6.4. Let $t \in\{1, \ldots, n\}$ and $M \in \mathbb{Z}$. Assume that all the Laurent monomials $c x_{1}^{r_{1}} \cdots x_{n}^{r_{n}}$ $(c \neq 0 \in \mathbb{Z} \mathbb{P})$ appearing in the sum $S:=\sum_{\mathbf{b}} u(\mathbf{b}) z[\mathbf{b}](u(\mathbf{b}) \in \mathbb{Z} \mathbb{P})$ satisfy $-r_{t} \leq M$. Then $\left\langle\mathbf{b}, e_{t}\right\rangle \leq M$ for all $\mathbf{b}$ with $u(\mathbf{b}) \neq 0$ in $S$.

Proof. We first assume that $r_{t} \leq 0$. Take the lexicographic order

$$
x_{t}>x_{t-1}>\cdots>x_{1}>x_{t+1}>x_{t+2}>\cdots>x_{n}>1 .
$$

Consider the map $f: \mathbb{Z}^{n} \rightarrow \mathbb{Z}^{n}$ that sends $\mathbf{b}$ to the exponent vector of the lowest term of $z[\mathbf{b}]$.

We claim that $f$ is a triangularizable coordinate-wise $\mathbb{Z}_{\geq 0}$-linear map by taking the order $(t, t-1, \ldots, 1, t+1, t+2, \ldots, n)$. Indeed, the proof is similar to the one of Lemma 6.2 , the only difference is that now we have

$$
f\left(e_{k}\right)= \begin{cases}-e_{k} \text { or }-e_{k}+\sum_{i<k} b_{i k} e_{i}, & \text { if } k<t, \\ -e_{k} \text { or }-e_{k}+\sum_{k<i \leq n}\left(-b_{i k}\right) e_{i}, & \text { if } k>t, \\ -e_{k}+\sum_{i<k} b_{i k} e_{i} \text { or }-e_{k}+\sum_{k<i \leq n}\left(-b_{i k}\right) e_{i}, & \text { if } k=t .\end{cases}
$$

(It is unnecessary for our purpose to figure out the exact value of $f\left(e_{k}\right)$.)

Thus we conclude that $f$ is bijective by Lemma 6.1 . Let $z\left[\mathbf{b}^{\prime}\right]$ be the one that appears in $S$ whose lowest Laurent monomial $c x_{t}^{-\left\langle\mathbf{b}^{\prime}, e_{t}\right\rangle} \prod_{i \neq t} x_{i}^{r_{i}}$ is smallest. Note that $\left\langle\mathbf{b}, e_{t}\right\rangle \leq\left\langle\mathbf{b}^{\prime}, e_{t}\right\rangle$ for all $\mathbf{b}$ appearing in $S$. By the injectivity of $f$, the lowest Laurent monomial $c x_{t}^{-\left\langle\mathbf{b}^{\prime}, e_{t}\right\rangle} \prod_{i \neq t} x_{i}^{r_{i}}$ will not cancel out with other terms in $S$. So $\left\langle\mathbf{b}^{\prime}, e_{t}\right\rangle \leq M$ by the assumption that all the Laurent monomials $c x_{1}^{r_{1}} \cdots x_{n}^{r_{n}}$ that appear in $S$ satisfy $-r_{t} \leq M$. Thus $\left\langle\mathbf{b}, e_{t}\right\rangle \leq M$ for all $\mathbf{b}$ appearing in $S$. 
Define a partial order $\prec$ on $\mathbb{Z}^{n}$ :

$$
\mathbf{b} \prec \mathbf{a} \Leftrightarrow b_{i} \leq a_{i} \text { for all } 1 \leq i \leq n, \quad \text { and } \quad \sum_{i}\left[b_{i}\right]_{+}<\sum_{i}\left[a_{i}\right]_{+} .
$$

Lemma 6.5. For each $\mathbf{a} \in \mathbb{Z}^{n}$, we have

$$
\tilde{x}[\mathbf{a}]-z[\mathbf{a}]=\sum_{\mathbf{b} \prec \mathbf{a}} u(\mathbf{a}, \mathbf{b}) z[\mathbf{b}], \quad \text { where } \quad u(\mathbf{a}, \mathbf{b}) \in \mathbb{Z} \mathbb{P} .
$$

Proof. Since $z[\mathbf{a}]$ form a $\mathbb{Z} \mathbb{P}$-basis of the cluster algebra $\mathcal{A}$, we can write

$$
\tilde{x}[\mathbf{a}]=\sum_{\mathbf{b}} u(\mathbf{a}, \mathbf{b}) z[\mathbf{b}]
$$

where $u(\mathbf{a}, \mathbf{b}) \neq 0 \in \mathbb{Z} \mathbb{P}$. For $1 \leq t \leq n$, it is easy to see that all the Laurent monomials $c x_{1}^{r_{1}} \cdots x_{n}^{r_{n}}(c \neq 0 \in \mathbb{Z} \mathbb{P})$ appeared $\tilde{x}[\mathbf{a}]$ satisfy $-r_{t} \leq a_{t}$, thus $b_{t} \leq a_{t}$ by Lemma 6.4. Together with Lemma 6.3 we can conclude that

$$
\tilde{x}[\mathbf{a}]-z[\mathbf{a}]=\sum_{\mathbf{b}} u(\mathbf{a}, \mathbf{b}) z[\mathbf{b}], \quad u(\mathbf{a}, \mathbf{b}) \in \mathbb{Z} \mathbb{P}
$$

where $\mathbf{b} \neq \mathbf{a}$ satisfies $b_{i} \leq a_{i}$ for all $1 \leq i \leq n$.

In the rest we show that $\sum_{i}\left[b_{i}\right]_{+}<\sum_{i}\left[a_{i}\right]_{+}$. For simplicity, we assume that the first $n^{\prime}$ coordinates of $\mathbf{a}$ are positive and the rest are non-positive. If $n^{\prime}=n$, then $\sum_{i}\left[b_{i}\right]_{+}<\sum_{i}\left[a_{i}\right]_{+}$ since $\mathbf{b} \neq \mathbf{a}$. Assume $n^{\prime}<n$. Regard $x_{i}$ for $n^{\prime}<i \leq n$ as frozen variable. Dividing (6.4) by the monomial $x_{n^{\prime}+1}^{-a_{n^{\prime}+1}} \cdots x_{n}^{-a_{n}}$, we get the expansion of

$$
\tilde{x}\left[\left(a_{1}, \ldots, a_{n^{\prime}}\right)\right]-z\left[\left(a_{1}, \ldots, a_{n^{\prime}}\right)\right]
$$

in the basis $\left\{z\left[\mathbf{b}^{\prime}\right]\right\}_{\mathbf{b} \in \mathbb{Z}^{n^{\prime}}}$ with coefficients in $\mathbb{Z} \mathbb{P}\left[x_{n^{\prime}+1}^{ \pm 1}, \ldots, x_{n}^{ \pm 1}\right]=\mathbb{Z}\left[x_{n^{\prime}+1}^{ \pm 1}, \ldots, x_{m}^{ \pm 1}\right]$. Using induction on $n$, we conclude that $\mathbf{b}^{\prime} \neq\left(a_{1}, \ldots, a_{n^{\prime}}\right)$, thus $\sum_{i}\left[b_{i}\right]_{+}<\sum_{i}\left[a_{i}\right]_{+}$.

Proof of Theorem 1.2. The $\mathbb{Z} \mathbb{P}$-independency of $\{\tilde{x}[\mathbf{a}]\}_{\mathbf{a} \in \mathbb{Z}^{n}}$ is asserted in Lemma 6.3. Now we show that the $\mathbb{Z P}$-linear span of $\{\tilde{x}[\mathbf{a}]\}_{\mathbf{a} \in \mathbb{Z}^{n}}$ equals $\mathcal{A}$. Since $\{z[\mathbf{a}]\}_{\mathbf{a} \in \mathbb{Z}^{n}}$ is a $\mathbb{Z P}$-basis of $\mathcal{A}$, it suffices to show that, for any $\mathbf{a} \in \mathbb{Z}^{n}, z[\mathbf{a}]$ can be expressed as a $\mathbb{Z} \mathbb{P}$-linear combination of $\tilde{x}\left[\mathbf{a}^{\prime}\right]$ for $\mathbf{a}^{\prime} \in \mathbb{Z}^{n}$. Iteratively applying Lemma 6.5 , and noting that $z[\mathbf{b}]=\tilde{x}[\mathbf{b}]$ for $\mathbf{b} \in \mathbb{Z}_{<0}^{n}$ (actually the equality holds if $\mathbf{b}$ has at most one positive coordinate), we can express $z[\mathbf{a}]$ as a linear combination of $\tilde{x}[\mathbf{b}]$ 's.

\subsection{The second proof}

Define a partial order on $\mathbb{Z}^{n}$ by

$$
\mathbf{b} \prec \mathbf{a} \text { if and only if } \sum_{i=1}^{n}\left[b_{i}\right]_{+}<\sum_{i=1}^{n}\left[a_{i}\right]_{+} \text {. }
$$

Definition 6.6. For $\mathbf{a} \in \mathbb{Z}^{n}$, let $T^{\mathbf{a}}$ be the set of all quasi-compatible collections and let $T_{\text {gcc }}^{\mathbf{a}} \subset T^{\mathbf{a}}$ be the globally compatible collections. (See Definition 5.4 for the terminology.) 
Let $\mathbf{a} \in\{0,1\}^{n}$. Let $C^{(i, j)}=\left\{u_{1}^{(i, j)}, v_{1}^{(i, j)}\right\}$ be the corner associated to the edge $(i, j)$ (if it exists). Let $\bar{T}^{\mathbf{a}}=T^{\mathbf{a}} \backslash T_{\mathrm{gcc}}^{\mathbf{a}}$ and let $t$ be a quasi-compatible collection in $\bar{T}^{\mathbf{a}}$. Let $c(t)$ be the set of all corners of $t$. We can define an equivalence relation on quasi-compatible collections $t, s \in \bar{T}^{\mathbf{a}}$ by $t \sim s$ if and only if $c(t)=c(s)$. Let $M_{t} \subset \bar{T}^{\mathbf{a}}$ denote the equivalence class of $t$ with respect to $\sim$. To each equivalence class we can associate a vector

$$
\mathbf{c}_{t}=\sum_{C^{(i, j)} \in c(t)}\left(\sum_{(i, h) \in Q_{B}} b_{i h} e_{h}+\sum_{(k, j) \in Q_{B}}\left(-b_{j k}\right) e_{k}\right) \in \mathbb{Z}_{\geq 0}^{n} .
$$

For each $M_{t} \subset \bar{T}^{\mathbf{a}}$ we also associate a vector $\mathbf{b}_{t}=\mathbf{a}-\mathbf{c}_{t}$. Note that $\mathbf{b}_{t} \prec \mathbf{a}$. Indeed, since $t \notin T_{\mathrm{gcc}}^{\mathbf{a}}$, there is at least one corner $C^{(i, j)}$ in $c(t)$, then $\left(\mathbf{b}_{t}\right)_{i} \leq a_{i}-\left(-b_{j i}\right)<a_{i}=1$, $\left(\mathbf{b}_{t}\right)_{j} \leq a_{j}-b_{i j}<a_{j}=1$, and $\left(\mathbf{b}_{t}\right)_{k} \leq a_{k}$ for all $k$, thus $\sum_{r=1}^{n}\left[\left(\mathbf{b}_{t}\right)_{r}\right]_{+}<\sum_{r=1}^{n}\left[a_{r}\right]_{+}$.

Let $\mathcal{D}_{\mathbf{a}}$ be the set of edges of Dyck paths for the vector $\mathbf{a}$. Let $\mathcal{D}_{\mathbf{b}_{t}}$ be defined analogously. Since $\mathbf{b}_{t} \prec \mathbf{a}, \mathcal{D}_{\mathbf{b}_{t}} \subset \mathcal{D}_{\mathbf{a}}$ (since each horizontal (resp. vertical) edge in $\mathcal{D}_{\mathbf{b}_{t}}$ naturally corresponds to a horizontal (resp. vertical) edge in $\mathcal{D}_{\mathbf{a}}$ ), and by the definition of $\mathbf{b}_{t}$ we have

$$
\mathcal{D}_{\mathbf{a}} \backslash \mathcal{D}_{\mathbf{b}_{t}}=\bigcup_{C^{(i, j)} \subset s \in M_{t}}\left\{v_{1}^{(k, j)}, u_{1}^{(i, h)}, u_{1}^{(j, f)}, v_{1}^{(g, i)} \mid(j, f),(g, i),(i, h),(k, j) \in Q_{\tilde{B}}\right\} .
$$

Definition 6.7. Define the map $\phi_{\mathbf{b}_{t}}: T^{\mathbf{b}_{t}} \rightarrow \bar{T}^{\mathbf{a}}$ as follows

$$
\phi_{\mathbf{b}_{t}}(d)=\left(\bigcup_{C^{(i, j)} \subset s \in M_{t}}\left\{u_{1}^{(i, h)}, v_{1}^{(k, j)} \mid(i, h),(k, j) \in Q_{\tilde{B}}\right\}\right) \sqcup d .
$$

It is easy to see that if $d \in T^{\mathbf{b}_{t}}$ is quasi-compatible then so is $\phi_{\mathbf{b}_{t}}(d) \in \bar{T}^{\mathbf{a}}$ and that $\phi_{\mathbf{b}_{t}}$ is injective.

Lemma 6.8. Let $\mathbf{a} \in\{0,1\}^{n}$, and $t, M_{t}, \mathbf{b}_{t}, \phi_{\mathbf{b}_{t}}$ be defined as above.

1. If there exists a quasi-compatible collection $d \in T^{\mathbf{b}_{t}}$ such that $\phi_{\mathbf{b}_{t}}(d) \in M_{t}$, then $M_{t}$ is contained in the image of $\phi_{\mathbf{b}_{t}}$. Furthermore, $\left\{\operatorname{Im} \phi_{\mathbf{b}_{t}} \mid M_{t} \subset \bar{T}^{\mathbf{a}}\right\}$ cover $\bar{T}^{\mathbf{a}}$.

2. For $A \subset T^{\mathbf{a}}$ define

$$
z[A]:=\left(\prod_{i=1}^{n} x_{i}^{-a_{i}}\right) \sum_{s \in A}\left(\prod_{(i, j) \in Q_{\tilde{B}}} x_{i}^{b_{i j}\left|S_{2}^{(i, j)}\right|} x_{j}^{-b_{j i}\left|S_{1}^{(i, j)}\right|}\right) .
$$

Then $z\left[\operatorname{Im} \phi_{\mathbf{b}_{t}}\right]=z\left[\mathbf{b}_{t}\right]$. It follows immediately that if $\operatorname{Im} \phi_{\mathbf{b}_{t}}=M_{t}$ then $z\left[M_{t}\right]$ is a standard monomial.

3. We can define a partial order on $\left\{M_{t}\right\}$ by inclusion of sets of corners. If $M_{t}$ is maximal with respect to this order then $\operatorname{Im} \phi_{\mathbf{b}_{t}}=M_{t}$.

Proof. (1) To see that the $\left\{\operatorname{Im} \phi_{\mathbf{b}_{t}}\right\}$ cover we will show that $M_{t} \subset \operatorname{Im} \phi_{\mathbf{b}_{t}}$. It suffices to find a single quasi-compatible collection that maps into $M_{t}$, because If $\phi_{\mathbf{b}_{t}}(d) \in M_{s}$ then any $w$ in $M_{s}$ is a disjoint union of

$$
\bigcup_{C^{(i, j)} \subset s \in M_{t}}\left\{v_{1}^{(k, j)}, u_{1}^{(i, h)} \mid(i, h),(k, j) \in Q_{\tilde{B}}\right\}
$$


with an element in $T^{\mathbf{b}_{t}}$. To produce this collection take a globally compatible collection in $T^{\mathbf{b}_{t}}$ that contains no $u_{1}^{(k, j)}$ or $v_{1}^{(i, h)}$ for all $i, j$ such that $C^{(i, j)} \subset t$ and for all $(k, j),(i, h) \in Q_{\tilde{B}}$. This property guarantees that no new corners besides those in $\mathbf{c}(t)$ will be created when we map it into $\bar{T}^{\mathbf{a}}$. Therefore its image under $\phi_{\mathbf{b}_{t}}$ is in $M_{t}$.

(2) Using the fact that every quasi-compatible collection in the image of $\phi_{\mathbf{b}_{t}}$ shares the edges

$$
\bigcup_{C^{(i, j)} \subset s \in M_{t}}\left\{v_{1}^{(k, j)}, u_{1}^{(i, h)} \mid(i, h),(k, j) \in Q_{\tilde{B}}\right\},
$$

and the definition of $\mathbf{b}_{t}$ it follows that

$$
\begin{aligned}
z\left[\mathbf{b}_{t}\right] & =\left(\prod_{i=1}^{n} x_{i}^{-\left(\mathbf{b}_{t}\right)_{i}}\right) \sum_{s \in T^{\mathbf{b}_{t}}}\left(\prod_{(i, j) \in Q_{\tilde{B}}} x_{i}^{b_{i j}\left|S_{2}^{(i, j)}\right|} x_{j}^{-b_{j i}\left|S_{1}^{(i, j)}\right|}\right) \\
& =\left(\prod_{i=1}^{n} x_{i}^{-a_{i}}\right)\left(\prod_{i=1}^{n} x_{i}^{\left(\mathbf{c}_{t}\right)_{i}}\right) \sum_{s \in T^{\mathbf{b}_{t}}}\left(\prod_{(i, j) \in Q_{\tilde{B}}} x_{i}^{b_{i j}\left|S_{2}^{(i, j)}\right|} x_{j}^{-b_{j i}\left|S_{1}^{(i, j)}\right|}\right) \\
& =\left(\prod_{i=1}^{n} x_{i}^{-a_{i}}\right) \sum_{s \in \operatorname{Im} \phi_{\mathbf{b}_{t}}}\left(\prod_{(i, j) \in Q_{\tilde{B}}} x_{i}^{b_{i j}\left|S_{2}^{(i, j)}\right|} x_{j}^{-b_{j i}\left|S_{1}^{(i, j)}\right|}\right)=z\left[\operatorname{Im} \phi_{\mathbf{b}_{t}}\right] .
\end{aligned}
$$

(3) We have already shown that $M_{t} \subset \operatorname{Im} \phi_{\mathbf{b}_{t}}$. Since $M_{t}$ is maximal, $\mathbf{c}(t)$ is a maximal set of corners and every element in the image of $\phi_{\mathbf{b}_{t}}$ contains $\mathbf{c}(t)$. So $\operatorname{Im} \phi_{\mathbf{b}_{t}} \subset M_{t}$.

We need the following facts about $z[\mathbf{a}]$.

Lemma 6.9. For any $\mathbf{a}, \mathbf{b} \in \mathbb{Z}^{n}$,

$$
z[\mathbf{a}] z[\mathbf{b}]=\sum_{\mathbf{c}} u(\mathbf{c}) z[\mathbf{c}], \quad u(\mathbf{c}) \in \mathbb{Z} \mathbb{P}
$$

where c satisfies $\left[c_{t}\right]_{+} \leq \sum\left[a_{t}+b_{t}\right]_{+}$for $1 \leq t \leq n$.

Proof. Regard $x_{i}(i \neq t)$ as frozen variables, i.e., $x_{t}$ as the only non-frozen variable. Then it is reduced to proving the lemma for $n=1$, which is easy.

Now we can prove the following lemma.

Lemma 6.10. For $\mathbf{a} \in \mathbb{Z}^{n}$ the expansion of $\tilde{x}[\mathbf{a}]$ in the basis of standard monomials is of the form

$$
\tilde{x}[\mathbf{a}]-z[\mathbf{a}]=\sum_{\mathbf{b} \prec \mathbf{a}} u(\mathbf{a}, \mathbf{b}) z[\mathbf{b}],
$$

where $u(\mathbf{a}, \mathbf{b}) \in \mathbb{Z} \mathbb{P}$ and all but finitely many are zero, and $\prec$ as is defined in (6.5).

Proof. Step I. We prove the case when $m=n$, i.e., $\mathbb{Z} \mathbb{P}=\mathbb{Z}$.

By the multiplicative property of $\{\tilde{x}[\mathbf{a}]\}$ and $\{z[\mathbf{a}]\}$ (Lemma 3.3) it is sufficient to show the result for $\mathbf{a} \in\{0,1\}^{n}$. Indeed, if $\mathbf{a}=\mathbf{a}^{\prime}+\mathbf{a}^{\prime \prime}$ and we have the result for $\mathbf{a}^{\prime}$, and $\mathbf{a}^{\prime \prime}$ then

$$
\begin{gathered}
\tilde{x}[\mathbf{a}]=\tilde{x}\left[\mathbf{a}^{\prime}\right] \tilde{x}\left[\mathbf{a}^{\prime \prime}\right]=\left(z\left[\mathbf{a}^{\prime}\right]+\sum_{\mathbf{b}^{\prime} \prec \mathbf{a}^{\prime}} u\left(\mathbf{a}^{\prime}, \mathbf{b}^{\prime}\right) z\left[\mathbf{b}^{\prime}\right]\right)\left(z\left[\mathbf{a}^{\prime \prime}\right]+\sum_{\mathbf{b}^{\prime \prime} \prec \mathbf{a}^{\prime \prime}} u\left(\mathbf{a}^{\prime \prime}, \mathbf{b}^{\prime \prime}\right) z\left[\mathbf{b}^{\prime \prime}\right]\right) \\
\stackrel{(*)}{=} z\left[\mathbf{a}^{\prime}\right] z\left[\mathbf{a}^{\prime \prime}\right]+\sum_{\mathbf{b} \prec \mathbf{a}^{\prime}+\mathbf{a}^{\prime \prime}} u(\mathbf{b}) z[\mathbf{b}]=z[\mathbf{a}]+\sum_{\mathbf{b} \prec \mathbf{a}} u(\mathbf{b}) z[\mathbf{b}],
\end{gathered}
$$

where $(*)$ uses Lemma 6.9. 
We have that

$$
z[\mathbf{a}]=z\left[T^{\mathbf{a}}\right]=z\left[T_{\mathrm{gcc}}^{\mathbf{a}}\right]+z\left[\bar{T}^{\mathbf{a}}\right]=\tilde{x}[\mathbf{a}]+z\left[\bar{T}^{\mathbf{a}}\right] .
$$

So we only need to write $z\left[\bar{T}^{\mathbf{a}}\right]$ in terms of standard monomials. Now $\left\{M_{t}\right\}$ forms a partition of $\bar{T}^{\mathrm{a}}$. So,

$$
\begin{aligned}
z\left[\bar{T}^{\mathbf{a}}\right] & =\left(\prod_{i=1}^{n} x_{i}^{-a_{i}}\right) \sum_{s \in \bar{T}^{\mathbf{a}}}\left(\prod_{(i, j) \in Q_{\tilde{B}}} x_{i}^{b_{i j}\left|S_{2}^{(i, j)}\right|} x_{j}^{-b_{j i}\left|S_{1}^{(i, j)}\right|}\right) \\
& =\left(\prod_{i=1}^{n} x_{i}^{-a_{i}}\right) \sum_{M_{t} \subset \bar{T}^{\mathbf{a}}} \sum_{s \in M_{t}}\left(\prod_{(i, j) \in Q_{\tilde{B}}} x_{i}^{b_{i j}\left|S_{2}^{(i, j)}\right|} x_{j}^{-b_{j i}\left|S_{1}^{(i, j)}\right|}\right)=\sum_{M_{t} \subset \bar{T}^{\mathbf{a}}} z\left[M_{t}\right] .
\end{aligned}
$$

Note that the $z\left[M_{t}\right]$ are not necessarily standard monomials. However by Lemma $6.8, z\left[\operatorname{Im} \phi_{\mathbf{b}_{t}}\right]$ are standard monomials, and $\left\{M_{t}\right\}$ partition the image of $\phi_{\mathbf{b}_{t}}$. Therefore we can do the same manipulation as above to get the equation

$$
z\left[\mathbf{b}_{t}\right]=z\left[\operatorname{Im} \phi_{\mathbf{b}_{t}}\right]=\sum_{M_{s} \subset \operatorname{Im} \phi_{\mathbf{b}_{t}}} z\left[M_{s}\right]
$$

Now there are finitely many $\mathbf{b}_{t}$ and $\mathbf{b}_{t} \prec a$ (since $\mathbf{c}_{t} \in \mathbb{Z}_{>0}^{n}$ ), so to finish the proof of our claim we only need to show that $z\left[\bar{T}^{a}\right]$ is a $\mathbb{Z}$-linear combination of these $z\left[\mathbf{b}_{t}\right]$ 's.

Note that $M_{s} \subset \operatorname{Im} \phi_{\mathbf{b}_{t}}$ has at least the corners of $c(t)$ but could have more. So $M_{s} \not \subset \operatorname{Im} \phi_{b_{t}}$ if $M_{s}<M_{t}$ with respect to our order in Lemma 6.8(3), and we can rewrite (6.6) as

$$
z\left[b_{t}\right]=z\left[M_{t}\right]+\sum_{M_{t}<M_{s}} d_{s} z\left[M_{s}\right], \quad d_{s} \in\{0,1\} .
$$

By extending the partial order on $\left\{M_{t}\right\}$ to a total order, we see that the transition matrix between $\left\{z\left[\mathbf{b}_{t}\right]\right\}$ and $\left\{z\left[M_{t}\right]\right\}$ is triangular with all diagonal entries 1 , so it is invertible over $\mathbb{Z}$. Therefore every $z\left[M_{t}\right]$ is a linear combination of $z\left[\mathbf{b}_{t}\right]$.

Therefore, $z\left[\bar{T}^{\mathbf{a}}\right]$ is a $\mathbb{Z}$-linear combination of $z\left[\mathbf{b}_{t}\right]$, where $t$ satisfies $M_{t} \in \bar{T}^{\mathbf{a}}$.

Step II. We prove the principal coefficient case, i.e., $m=2 n$ and $\tilde{B}=\left[\begin{array}{c}B \\ I_{n}\end{array}\right]$ where $I_{n}$ is the $n \times n$ identity matrix.

Let $\mathbb{Z} \mathbb{P}=\mathbb{Z}\left[y_{1}, \ldots, y_{n}\right]$ where $y_{j}=x_{n+j}$ for $1 \leq j \leq n$. Note that this will imply the general coefficient case by replacing the principal coefficients $y_{j}$ by $\prod_{i=n+1}^{m} x_{i}^{b_{i j}}$ for $1 \leq j \leq n$.

Apply Step I to the coefficient-free cluster algebra $\mathcal{A}^{\prime}$ with $B$-matrix $\left[\begin{array}{cc}B & -I_{n} \\ I_{n} & 0\end{array}\right]$. For any $\mathbf{a} \in \mathbb{Z}^{n}$, denote $\tilde{\mathbf{a}}=\left(a_{1}, \ldots, a_{n}, 0, \ldots, 0\right) \in \mathbb{Z}^{2 n}$. Then $\tilde{x}[\tilde{\mathbf{a}}]=\tilde{x}[\mathbf{a}]$ and $z[\tilde{\mathbf{a}}]=z[\mathbf{a}]$. Thus

$$
\tilde{x}[\mathbf{a}]-z[\mathbf{a}]=\tilde{x}[\tilde{\mathbf{a}}]-z[\tilde{\mathbf{a}}]=\sum u\left(\mathbf{b}^{\prime}\right) z\left[\mathbf{b}^{\prime}\right],
$$

where $u\left(\mathbf{b}^{\prime}\right) \in \mathbb{Z}$ and $\mathbf{b}^{\prime}=\left(b_{1}, \ldots, b_{2 n}\right)$ satisfies $\sum_{i=1}^{m}\left[b_{i}\right]_{+}<\sum_{i=1}^{n}\left[a_{i}\right]_{+}$. So it suffices to show that $z\left[\mathbf{b}^{\prime}\right]$ is a $\mathbb{Z} \mathbb{P}$-linear combination of $z[\mathbf{c}]$ with $\mathbf{c}=\left(c_{1}, \ldots, c_{n}\right) \in \mathbb{Z}^{n}$ satisfying $\sum_{i=1}^{n}\left[c_{i}\right]_{+} \leq \sum_{i=1}^{n}\left[b_{i}\right]_{+}$ (so $\left.\sum_{i=1}^{n}\left[c_{i}\right]_{+} \leq \sum_{i=1}^{m}\left[b_{i}\right]_{+}<\sum_{i=1}^{n}\left[a_{i}\right]_{+}\right)$.

Denote $\mathbf{b}=\left(b_{1}, \ldots, b_{n}\right)$. Since $z\left[\mathbf{b}^{\prime}\right]=z[\mathbf{b}] z\left[\left(0, \ldots, 0, b_{n+1}, \ldots, b_{2 n}\right)\right]$ where the second factor is a $\mathbb{Z P}$-linear combination of $z[\mathbf{d}]$ with $\mathbf{d}=\left(d_{1}, \ldots, d_{n}\right) \in \mathbb{Z}_{\leq 0}^{n}$, it suffices to show that $z[\mathbf{b}] z[\mathbf{d}]$ 
is a $\mathbb{Z P}$-linear combination of $z[\mathbf{c}]$ with $\mathbf{c}=\left(c_{1}, \ldots, c_{n}\right) \in \mathbb{Z}^{n}$ satisfying $\sum\left[c_{i}\right]_{+} \leq \sum\left[b_{i}\right]_{+}$. This follows from Lemma 6.9 since $\sum_{i=1}^{n}\left[c_{i}\right]_{+} \leq \sum_{i=1}^{n}\left[b_{i}+d_{i}\right]_{+} \leq \sum_{i=1}^{n}\left[b_{i}\right]_{+}$.

Proof of Theorem 1.2. Similar to the proof in Section 6.1, except that we use Lemma 6.10 in place of Lemma 6.5 .

\section{Acknowledgements}

The authors are grateful to the anonymous referees for carefully reading through the manuscript and giving us many constructive suggestions to improve the presentation. KL is supported by Wayne State University, Korea Institute for Advanced Study, AMS Centennial Fellowship and NSA grant H98230-14-1-0323. MM is supported by GAANN Fellowship.

\section{References}

[1] Bayleran D.E., Finnigan D.J., Haj Ali A., Lee K., Locricchio C.M., Mills M.R., Stiefel D.P.P., Tran T.M., Urbaniuk R., A new combinatorial formula for cluster monomials of equioriented type $A$ quivers, Preprint, 2014, available at http://math.wayne.edu/ klee/Cluster_A_public.pdf.

[2] Beineke A., Brüstle T., Hille L., Cluster-cylic quivers with three vertices and the Markov equation (with an appendix by Otto Kerner), Algebr. Represent. Theory 14 (2011), 97-112, math.RA/0612213.

[3] Benito A., Muller G., Rajchgot J., Smith K., Singularities of locally acyclic cluster algebras, arXiv:1404.4399.

[4] Berenstein A., Fomin S., Zelevinsky A., Cluster algebras. III. Upper bounds and double Bruhat cells, Duke Math. J. 126 (2005), 1-52, math.RT/0305434.

[5] Fomin S., Zelevinsky A., Cluster algebras. I. Foundations, J. Amer. Math. Soc. 15 (2002), 497-529, math.RT/0104151.

[6] Grabowski J., Graded cluster algebras, arXiv:1309.6170.

[7] Gross M., Hacking P., Keel S., Birational geometry of cluster algebras, Algebr. Geom. 2 (2015), 137-175, arXiv:1309.2573.

[8] Gross M., Hacking P., Keel S., Kontsevich M., Canonical bases for cluster algebras, arXiv:1411.1394.

[9] Lee K., Li L., Zelevinsky A., Greedy elements in rank 2 cluster algebras, Selecta Math. (N.S.) 20 (2014), 57-82, arXiv:1208.2391.

[10] Lee K., Schiffler R., Positivity of cluster algebras, Ann. of Math. 182 (2015), 73-125, arXiv:1306.2415.

[11] Matherne J.P., Muller G., Computing upper cluster algebras, Int. Math. Res. Not. 2015 (2015), 3121-3149, arXiv:1307.0579.

[12] Muller G., $\mathcal{A}=\mathcal{U}$ for locally acyclic cluster algebras, SIGMA 10 (2014), 094, 8 pages, arXiv:1308.1141.

[13] Plamondon P.G., Generic bases for cluster algebras from the cluster category, Int. Math. Res. Not. 2013 (2013), 2368-2420, arXiv:1308.1141.

[14] Plamondon P.G., Private communication, 2014.

[15] Seven A.I., Maximal green sequences of skew-symmetrizable $3 \times 3$ matrices, Linear Algebra Appl. 440 (2014), 125-130, arXiv:1207.6265.

[16] Speyer D., An infinitely generated upper cluster algebra, arXiv:1305.6867. 